\title{
NONLINEAR SATURATION OF THE TRAPPED-ION MODE BY MODE COUPLING \\ IN TWO DIMENSIONS
}

BRUCE I, COHEN AND W, M. TANG

\section{PLASMA PHYSICS LABORATORY}

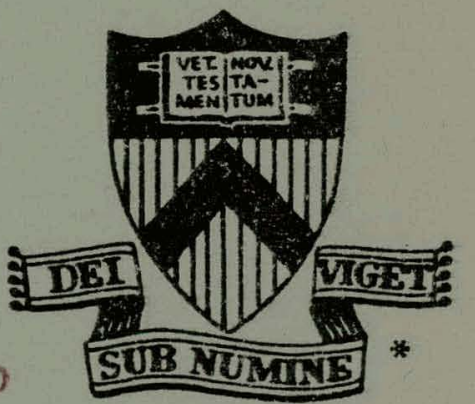

\section{PRINCETON \\ UNIVERSITY PRINCETON, NEW JERSEY}

This work was supported by 0 . S. Energy Research and Development Administration Contract EY-76-C-02-3073. Reproduction, translation, publication, use and disposal, in whole or in part, by or for the United States Government is permitted. 


\section{DISCLAIMER}

This report was prepared as an account of work sponsored by an agency of the United States Government. Neither the United States Government nor any agency Thereof, nor any of their employees, makes any warranty, express or implied, or assumes any legal liability or responsibility for the accuracy, completeness, or usefulness of any information, apparatus, product, or process disclosed, or represents that its use would not infringe privately owned rights. Reference herein to any specific commercial product, process, or service by trade name, trademark, manufacturer, or otherwise does not necessarily constitute or imply its endorsement, recommendation, or favoring by the United States Government or any agency thereof. The views and opinions of authors expressed herein do not necessarily state or reflect those of the United States Government or any agency thereof. 


\section{DISCLAIMER}

Portions of this document may be illegible in electronic image products. Images are produced from the best available original document. 
NOTICE

This report was prepared as an account of work sponsored by the United States Government. Neither the United states nor the United States Energy Research and Development Administration, nor any of their employees, nor any of their contractors, subcontractors, or their employees, makes any warranty, express or implied, or assumes any legal liability or responsibility for the accuracy, completeness or usefulness of any information, apparatus, product or process disclosed, or represents that its use would not infringe privately owned rights.

Printed in the United States of America.

Available from

Natiorial Tecinical Information Service

U. S. Department of Commerce 5285 Port Royal Road Springfield, Virginia 22151

Price: Printed Copy \$_*; Microfiche $\$ 3.00$

*Pages

$1-50$

$51-150$

$151-325$

$326-500$

$501-1.000$
NTIS

Selling Price

$\$ 4.00$

5.45

7.60

10.60

13.60 
Nonlinear Saturation of the Trapped-Ion Mode by Mode

Coupling in Two Dimensions

\author{
Bruce I. Cohen \\ University of California \\ Lawrence Livermore Laboratory* \\ Livermore, CA 94550 \\ and \\ Princeton University \\ Plasma Physics Laboratory \\ Princeton, NJ 08540
}

and

\author{
W. M. Tang \\ Princeton University \\ Plasma Physics Laboratory \\ Princeton, NJ 08540
}

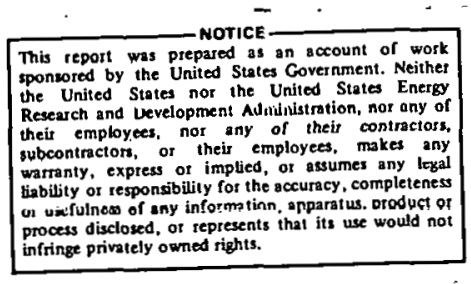

*Work performed under the auspices of the U.S. Energy Research and Development Administration under contract No. W-7405-Eng-48. 


\section{ABSTRACT}

A study of the nonlinear saturation by mode coupling of the dissipative trapped-ion mode is presented in which both radial and poloidal variations are considered. The saturation mechanism consists of the nonlinear coupling via $\underline{E} \times \underline{B}$ convection of energy from linearly unstable modes to stable modes. Stabilization is provided at short poloidal wavelengths by Landau damping from trapped and circulating ions, at short radial wavelengths by effects associated with the finite ion banana excursions and at long wavelengths by ion collisions. A one-dimensional, nonlinear partial differential equation for the electrostatic potential derived in earlier work is extended to two dimensions and to third order in amplitude. Included systematically are kinetic effects, e.g. Landau damping and its spatial dependence due to magnetic shear. The stability and accessibility of equilibria are considered in detail for cases far from as well as close to marginal stability. In the first case three-wave inter-

actions are found to be important when the spectrum of unstable modes is sufficiently narrow. In the latter case, it is found that for a single unstable mode, a four-wave interaction can provide the dominant saturation mechanism. Cross-field transport is calculated, and the scaling of results is considered for tokamak parameters. 


\section{Introduction}

It is well known that microinstabilities involving magnetically trapped particles can pose a potentially serious obstacle to efficient plasma confinement in toroidal systems [1-12]. Present tokamaks, such as T-10 and the Princeton Large Torus (PLT), are expected to achieve high enough temperatures

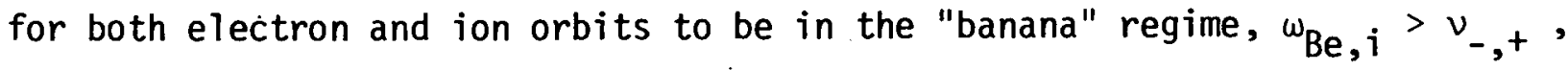
where $\omega_{\mathrm{Be}, i}$ are the trapped-electron and ion bounce frequencies and $\nu_{-,+}$are the effective electron and ion collision frequencies. For mode frequencies, $\omega$, below $\omega_{\mathrm{Bi}}$; it is predicted that electron collisions can drive drift waves unstable [1-12]. This so-called trapped-ion mode can lead to anomalously large transport $[11,12]$ and has motivated theoretical study of its 1inear [1-8] and nonlinear [9-16] properties. This paper considers mode coupling as a saturation mechanism and extends two earlier one-dimensional studies $[10,15]$. We will make frequent reference to these earlier studies, but will review some of their contents in an effort to make the present paper reasonably selfcontained.

The conventional description of the dissipative trapped-ion mode is that of a low frequency, quasineutral, electrostatic drift wave propagating in the electron diamagnetic drift direction. Electron collisions are destab1lizing whlle 1on collisions $[1,4]$ and Landau resonances with both circulating $[3,4]$ and trapped [5] ions are stabilizing. Most of the theoretical treatments cited do not address the issue of the radial mode structure, i.e. the mode is assumed to be localized in a region small relative to the plasma radius but large relative to the banana width. In studies of the nonlocal, linear radial problem $[17,6]$ it was found that shear exerts a stabilizing influence. We adopt a point of view similar to that of Gladd and Ross [6], i.e. all modes are assumed to have flute-like structure along the field lines 
$\left[0 \leqslant k_{11} \leqslant \mathcal{O}^{\prime}(\text { connection length })^{-1}\right.$. $]$. In treating the two-dimensional non mode structure orthogonal to the magnetic field, we allow for the presence of many mode rational surfaces [where $k_{11}(r)=0$ ].

The object of this study is to trace the nonlinear evolution of linearly unstable modes which undergo wave steepening as the result of $\underline{E} \times \underline{B}$ convection $[11,12]$. Kadomtsev and Pogutse have estimated a diffusion coefficient at saturation which is widely accepted as an upper limit, $D=\gamma / k^{2}(\gamma$ is the linear growth rate of the fastest growing mode with wavenumber $k$ ). Their physical arguments give a level of 'turbulence at saturation equivalent to that sufficient for local denşity fluctuations to have gradients comparable to the background gradient. LaQuey, Mahajan, Rutherford, and Tang (LMRT)[10] investigated the Kadomtsev and Pogutse slab model [11,12], and demonstrated that the instability can be saturated by phase-coherent, nonlinear $\underline{E} \times \underline{B}$ coupling of energy from unstable long:-wavelength modes to short-wavelength modes which are stabilized by Landau damping arising from trapped-ion bounce resonances for weak temperature gradients: $\eta_{i} \equiv d \ln \left(T_{j}\right) / d \ln \left(n_{0}\right)<2 / 3$. These kinetic effects were heuristically introduced into the fluid model. In the radially local limit far from mode rational surfaces, LMRT found coherent saturated states composed of many Fourier modes. Cohen; Krommes, Tang, and Rosenbluth [15] extended this study by determining the stability and accessibility of various nonlinear equilibria admitted by the LMRT model and by examining the influence of linear dispersion and ion collisions. Saisson and Wimmel [16] have considered the evolution of the Kadomtsev-Pogutse fluid equations by means of direct numerical integration. In the absence of Landau damping effects and ignoring wave steepening along the field line, they observe a saturation which occurs due to profile modification, but at substantially higher levels of turbulence as compared to the Kadomtsev-Pogutse estịmate. 
The present study deals with a two-dimensional analysis of mode coupling as a saturation mechanism for the trapped-ion mode. In particular, now we include the radially nonlocal effects of magnetic shear and hence many mode rational surfaces. Nonlinear terms arising from $\underline{E} \times \underline{B}$ convection and depending explicitly on radial derivatives are also taken into account. We present a more fundamental derivation of the nonlinear equation governing the evolution of the instability extended to $\boldsymbol{\mathcal { O }}(\mathrm{e} \phi / T)^{3}$. The derivation begins as in Ref. 10 with the Kadomtsev and Pogutse fluid equations. Important kinetic effects are systematically added to the quasineutrality condition in a perturbative fashion. When linearized, our fundamental equation is consistent with the results of a more exact kinetic theory describing the two-dimensional 1 inear mode structure of the trapped-ion mode [6].

In the interest of simplicity many potentially important effects are not considered here, e.g. ellipticity of the torus [5], toroidal gradient drifts $[7,18]$, impurities [8] and detrapping $[9,13,19]$. We will establish the parameter regimes in which approximations within our model are self-consistent and describe the scaling of our results with tokamak parameters. A more general analysis with a wider range of applicability demands a fully kinetic nonlinear treatment wilh a redistic coll1sion operator. This formidable calculation remains an outstanding problem.

The remainder of the paper is organized as follows. In Section 2 a derivation of the nonlinear model equation is reviewed in which a prescription is given for the systematic addition to the Kadomtsev-Pogutse fluid equations of weakly perturbative kinetic effects. In Section 3 the radially local model described in Refs. 10 and 15 is extended to include nonlinear $\underline{E} \times \underline{B}$ effects depending on radial derivatives of the potential. These effects are found to destabilize the radially uniform equilibria previously found to 
be stable to perturbations parallel to the propagation direction of the drift waves [15]. This motivates construction of new equilibria with hitherto ignored linear and nonlinear, radially dependent physical effects included. These equilibria are shown to achieve much lower levels of saturated amplitude provided the waves are not too dispersive. This constraint on dispersion is necessary for the three-wave interactions to remain effective. Section 4 considers the case very close to linear marginal stability where a single unstable mode couples with itself by means of a four-wave interaction. A noni inear frequency shift is induced which allows a balance between linear frequencydependent driving and damping forces. In Section 5 the transport of particle flux is calculated and the scalings of the transport coefficient and the saturated amplitude e $\phi / T$ with tokamak parameters are examined. Section 5 concludes with a brief summary of the conditions under which our model is applicable and the principal results of our analysis.

\section{Muder Equaliuns}

In this section we present a derivation of the nonlinear equations needed to generalize the analysis of Ref. 15. Once again we use the Kadomtsev-Pogutse fluid equations $[11,12]$ as a starting point. This model utilizes a four-fluid description consisting of circulating and trapped ions and electrons. In some sense an average has been taken over the bounce motion of the trapped particles. This isi justified because the mode frequency is much smaller than the bounce frequencies $\omega \ll \omega_{B i}$, e where $\omega_{B S} \equiv\left(\varepsilon T_{S} / 2 m_{S}\right)^{1 / 2} / q R, q$ is the safety factor, $\varepsilon=r / R$ is the inverse aspect ratio, and $R$ is the major radius of the torus.

We consider the familiar slab coordinate system (Fig. 1) with uniform magnetic field in the $z$ direction, density and temperature gradients in the $x$ (radial) direction, and the diamagnetic drift therefore in the $y$ direction 
$\left[y=r\left(\theta-r_{5} / q\right)\right.$, where $\theta$ and $\zeta$ are the poloidal and toroidal angles $]$ : The intrinsic inhomogeneity of the tokamak magnetic field, which is proportional to $\varepsilon$, leads to a relative fraction $\varepsilon^{1 / 2}$ of magnetically trapped particles. Because the phase velocity of these modes lies below the thermal velocity of the ions, free streaming along the magnetic field lines causes the bulk of the circulating ions and electrons to respond adiabatically to the wave. The number densities here are given respectively by the quasi-steady Boltzmann distributions $\left(1-\varepsilon^{1 / 2}\right) n_{0} \exp ( \pm e \phi / T)$ for $T_{e}=T_{i} \equiv T$ and singly charged species. In evaluating the Boltzmann factors we have made a specific choice of gauge: $\int d^{3} \underline{x} \phi(\underline{x})=0$.

The effective collision frequencies are enhanced $\left(\nu_{-}=\nu_{e} / \varepsilon\right.$ and $\left.\nu_{+}=\nu_{j} / \varepsilon\right)$ because small-angle collisions are the dominant process for scattering particles into and out of the loss cone $\left(v_{11}>\varepsilon^{1 / 2} v_{1}\right)$ [12]. Collisions tend to relax the trapped particle densities to Boltzmann distributions $n_{e, i}^{T} \sim \varepsilon^{1 / 2} n_{0} \exp ( \pm e \phi / T)$ over characteristic times $\nu_{\bar{\tau}}^{-1}$. Using the simplest Krook model operator to treat collisions, we find that the momentum equations become

$$
n_{s^{\prime}} m_{s}\left[(\partial / \partial t)+\underline{v}_{s} \cdot \underline{\nabla}\right] \underline{v}=n_{s} e_{s}\left(-\underline{\nabla} \phi+\underline{v}_{s} \times \underline{B}^{-1}\right)-\underline{\nabla} \cdot \underline{p}_{s}+n_{s^{\prime}} m_{s} \sum_{s^{\prime}} v_{s s^{\prime}}\left(\underline{v}_{s^{\prime}}-\underline{v}_{s}\right),
$$

where $s$ denotes the species, ${ }_{=} s$ is the pressure tensor, $e_{s}$ is the charge, $n_{s}$ is the number density, $v_{s}$ is the fluid velocity, and $v_{s s^{\prime}}$ is the relative collision frequency. Temperature perturbations are consistently neglected. The usual orderings are assumed: $\nu_{+} \ll \omega \ll \nu_{-}, \omega_{B} i \ll \Omega_{e, j}$ where $\Omega_{e, j}$ are the cyclotron frequencies. We take the pressure tensor to be isotropic perpendirular to the magnetic field, which allows $\underline{\nabla} \cdot \underline{\underline{p}}_{S}+\nabla \mathrm{p}_{S} \equiv \underline{\nabla}\left(\mathrm{n}_{\mathrm{s}} \mathrm{T}\right)$ since $\partial / \partial z \equiv 0$. Eq. (1) is then solved perturbatively by expanding $n_{s}$ and $v_{s}$ in power series as functions of $\Phi \equiv e_{\phi} / T \ll 1$. 


$$
-6-
$$

The lowest order solution of Eq: (1) is given dominantly by 1 $v_{s}^{(0)} \approx \hat{y} c T / e_{s}: r_{p}$ where $r_{p} \equiv\left[d \ln \left(n_{0} T\right) d x\right]^{-1}$, and to first order in $\Phi$ by

$$
\underline{v}_{S}^{(1)} \approx \hat{c z} \times\left[\underline{\nabla} \phi+(\underline{\nabla} p / n e)_{s}^{(1)}\right] B^{-1}
$$

The polarization drift is smaller by $\left(\omega / \Omega_{s}\right)$, and the drift due to collisional drag is smaller by $\mathcal{O}\left(\dot{\psi}_{s} / \Omega_{s}\right)$. These as well as the gradient and curvature drifts are ignored [7]. The most important effect of weak magnetic shear appears in the untrapped ion response $[6,17]$ and demands a kinetic description which we discuss later in this section. At second order in $\Phi$,

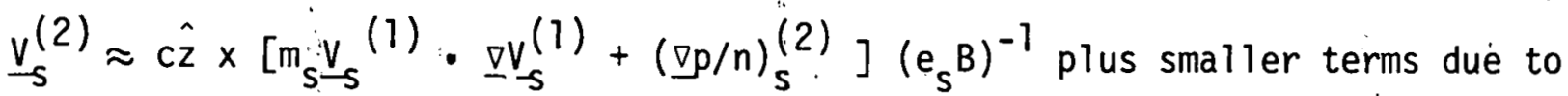
polarization effects and drag. Hence $\left|V_{s}^{(2)}\right| \sim \mathcal{O}\left(\mathrm{kV}_{\mathrm{s}}^{(1)} / \Omega_{\mathrm{s}}\right) V_{\mathrm{s}}^{(1)}$. Similarly, $\underline{v}_{s}^{(3)} \approx c \hat{z} \times\left[m_{s} \underline{v}_{s}^{(1)} \cdot \nabla \underline{v}_{s}^{(2)}+m_{s} \underline{v}_{s}^{(2)} \cdot \nabla_{-s}^{(1)}+(\underline{\nabla p} / n)_{\hat{B}}^{(3)}\right]\left(e_{s} B\right)^{-1} ;$ and $\left|v_{s}^{(3)}\right| \sim Q\left(k v_{s}^{(1)} / \Omega_{s}\right)^{2} v_{s}^{(1)}$. We have now evaluated the fluid velocities to $\left.d()^{3}\right)^{3}, \underline{v}_{s} \approx \underline{v}_{-}^{(0)}+\underline{v}_{s}^{(1)}+\underline{v}_{s}^{(2)}+\underline{v}_{s}^{(3)}$, and can replace the fluid velocities appearing in the continuity equations which follow with explicit functions of $\Phi$ and $n_{s}$.

The trapped particles satisfy continuity equations

$\partial n_{e, i}^{\top} / \partial t+\underline{\nabla} \cdot\left(n_{e, j}^{\top} \underline{v}_{e, i}^{\top}\right)=-v_{-,+}\left[n_{e, i}^{\top} \varepsilon^{l / 2} n_{0} \exp ( \pm e \Phi / T)\right]$

Because $\nu_{-} \gg \omega$ the trapped-electron density is dominantly Boltzmann-like $n_{e}^{\top} \approx \varepsilon^{1 / 2} n_{0} \exp (e \Phi / T)$. This is not the case for the ions for which $\nu_{+} \ll \omega$. closure of the fluid equations is obtained by invoking quasi-neutrality.

$n_{e}^{\top}+\left(1-\varepsilon^{1 / 2}\right) n_{0} \exp (e \phi / T)=n_{i}^{\top}+\left(1-\varepsilon^{1 / 2}\right) n_{0} \exp (-e \phi / T)+\delta n(\phi)$,

where $\delta n(\phi)$ is defined as the number density induced by the electrostatic 
potential not included in the foregoing fluid model. It is at this point that we systematically add linear kinetic effects not otherwise contained in the Kadomtsev-Pogutse mode, e.g. Landau damping, finite banana-width excursions, and thermal effects which contribute to linear dispersion. In general, the term on is the difference of the additional ion and electron contributions, $\delta n=\delta n_{j}-\delta n_{e}$

To determine $\delta$ we must construct the linear susceptibility $x_{s}(\omega, \underline{k})$ for species $s$ which is defined by the linear relation between the Fourier-Laplace transformed number density $\tilde{n}_{s}(\omega, k)$ and electrostatic potential $\tilde{\phi}(\omega, \underline{k})$ :

$$
\tilde{n}_{s}(\omega, \underline{k}) \equiv-k^{2} x_{s}(\omega, \underline{k}) \tilde{\phi}(\omega, \underline{k}) / 4 \pi e_{s} .
$$

Application of Eq. (5) to Poisson's equation and use of a kinetic equation to evaluate $x_{s}(\omega, \underline{k})$ lead to a linear dispersion relation for an electrostatic mode. For the trapped-ion mode in simplest approximation this can be expressed as [6]

$$
\begin{aligned}
0=\hat{\varepsilon}(\omega, \underline{k}) \equiv 1 & +\sum_{S} x_{S}(\omega, \underline{k})=1+\left(k \lambda_{e}\right)^{-2}\left\{2+\left\langle\frac{\varepsilon^{1 / 2}\left(\omega_{e}^{\star}-\omega\right)}{\omega-\omega D e+i \nu_{-}} T_{T}\right.\right. \\
& +\left\langle\frac{\varepsilon^{1 / 2}\left(\omega_{i}^{*}-\omega\right)(1-b)}{\omega-\omega_{D i}+i \nu_{+}}>+\left\langle\frac{\omega_{i}^{*}-\omega}{\omega-k_{11} V_{11}+i v_{i}}>U\right\},\right.
\end{aligned}
$$

where $\omega_{e, i}^{*}$ are the diamagnetic frequencies, $\omega_{D e, i}$ are the Doppler shifts arising from the curvature drifts, $b \equiv\left(k_{x}{ }^{2}+k_{y}{ }^{2}\right) \rho_{i}{ }^{2} q^{2} / \varepsilon \ll 1$, which gives the lowest order banana-width effects, $\rho_{i}$ is the ion gyro-radius, $\lambda_{e} \equiv\left(T / 4 \pi n_{0} e^{2}\right)^{l / 2}$ is the Debye length, and the subscripted brackets imply that there is an integration over the inagnetically trapped $(T)$ or untrapped (U) region of velocity space. In dealing with the ion banana-width correction term $(1-b)$, the simplest harmonic time dependence of the excursions of the trapped ions in the 
$x-y$ plane away from the magnetic field line is assumed [6].

In the usual fluid limit $\nu_{-} \gg \omega>\nu_{+}, \omega D_{e, j} \rightarrow 0, b<1$ and $k^{2} \lambda_{e}{ }^{2}<<1$, Eq. (6) gives after the velocity-space averaging

$$
\hat{\varepsilon}(\omega, \underline{k}) \approx\left(k \lambda_{e}\right)^{-2}\left[2+\frac{\varepsilon^{1 / 2} \omega_{e}^{*}}{i \nu_{-}}-\frac{\varepsilon^{1 / 2} \omega_{e}^{*}}{\omega+i v_{+}}\right] \approx 0,
$$

where $\omega_{e}^{*}=-\omega_{j}^{*}=k_{y} c T / e B r_{n}, r_{n} \equiv-\left[d_{i} \ln \left(n_{0}\right) / d x\right]^{-1}$ and $\nu_{ \pm}$are the effective ion and electron collision frequencies. This gives the same dispersion relation as produced earlier by the Kadomitsev-Pogutse fluid equations $[1,10]$, $\omega \approx \varepsilon^{7 / 2} \omega_{\mathrm{e}}^{*} / 2+i\left[\left(\varepsilon^{1 / 2} \omega_{\mathrm{e}}^{* / 2}\right)^{2} \nu_{-}^{-l}-\nu_{+}\right]$. Our prescription for including small kinetic corrections to the linear normal mode frequency is then to. use for the Fourier-Laplace transform of the number density sn the expression $\tilde{\delta}(\omega, \underline{k})=-k^{2} \delta_{x}(\omega, \underline{k}) \tilde{\phi}(\omega, \underline{k}) / 4 \pi e$, where $\delta x$ is obtained from the difference of Eqs. (6) and (7). We formally perform the inverse Fourier-Laplace transforms as follows:

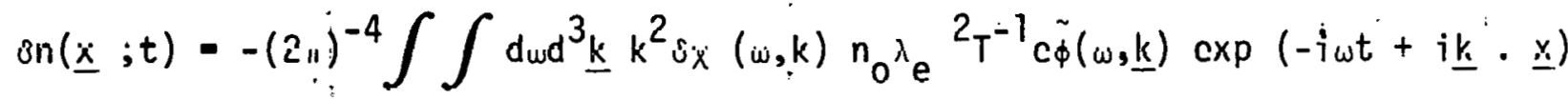

$$
\begin{aligned}
& \equiv \nabla^{2} \delta x(\underline{i} \partial / \partial t,-\underline{i} \underline{\nabla}) n_{0} \lambda^{2} \Phi(\underline{x} ; t)
\end{aligned}
$$

To illustrate the prescription for calculating $\delta n(\underline{x} ; t)$ we consider the kinetic contributions to the ion response arising from the finite ion bananawidth excursions, resonant and nonresonant untrapped ions, and resonant trapped ions. For the banana-width excursion contribution to the susceptibility, $\delta x_{B}$, we obtain from Eq. (6)

$$
k^{2} \lambda_{e}^{2} \delta x_{B}(\omega, \underline{k}) \approx\left(k_{x}^{2}+k_{y}^{2}\right)<\frac{\varepsilon^{1 / 2} \omega_{e}^{*}\left(\rho_{i}^{2} q^{2} / \varepsilon\right)}{\omega+i \nu+}>T
$$


Use of Eq. (8), the usual frequeney orderings, and $k r_{n} \gg>$ give

$$
\delta n_{B}(\underline{x} ; t) \approx-2 n_{0}\left(\rho_{i}{ }^{2} q^{2} / \varepsilon\right)\left(\partial^{2} / \partial x^{2}+\partial^{2} / \partial y^{2}\right)(\partial / \partial t)^{-1}\left(v_{\star}^{T} \partial / \partial y\right) \Phi(\underline{x} ; t),
$$

where $V_{\star}^{T} \equiv\left(\varepsilon^{1 / 2} / 2\right) c T / e B r$ is the electron diamagnetic drift velocity reduced by the factor $\varepsilon^{1 / 2} / 2$. The bounce resonances of the ions lead to dissipation $[4,5]$ :

$$
k^{2} \lambda_{e}^{2} \delta x_{T}(\omega, \underline{k}) \approx i 2 A^{\prime}(1-3 n / 2)<\frac{\omega^{4} / \omega_{B i}^{3}}{\omega+i \nu_{+}}>_{T}
$$

where $n \equiv d e n T / d$ en $n_{0}$ and $A^{\prime} \sim \sigma(20)$, from which it follows that $\delta n_{T}(\underline{x} ; t) \approx-2 n_{0} A^{\prime}(1-3 n / 2) \omega_{B i}{ }^{-3}\left(\partial^{4} / \partial t^{4}\right)(\partial / \partial t)^{-1} \Phi(\underline{x} ; t)$.

To insure Landau damping, $n<2 / 3$. We ignore dispersion effects associated with the trapped ions [5].

Important effects due to magnetic shear arise in the untrapped ion response because of the dependence of the resonance $\omega=k_{11} v_{11}$ upon the q-profile, i.e. effectively $k_{11}=|\ell q-m| / q R$, where $m$ is the poloidal mode number and $\ell$ is the toroidal mode number and integer minimizing $|e q-m|$ [6]. The locations of the mode rational surfaces are determined by $k_{11}=0$. Following the analysis of Gladd and Ross [6], the untrapped ion response is, in the limits of negligibly small untrapped ion collision frequency and untrapped bananawidth excursion,

$$
\begin{aligned}
\left\langle\frac{\omega_{i}^{*}-\omega}{\omega-k_{11} V_{11}}\right\rangle & =\frac{\omega_{i}^{*}-\omega}{\omega} \varepsilon^{1 / 2} z \cdot\left\{z(z)-z\left(\varepsilon^{1 / 2} z\right)+\frac{\omega_{j}^{\star} n}{\omega_{i}^{\star}-\omega}\right. \\
& \left.\times\left[z\left(1-\varepsilon^{1 / 2}\right)+\left(z^{2}-1 / 2\right) z(z)-\left(\varepsilon z^{2}-1 / 2\right) z\left(\varepsilon^{1 / 2} z\right)\right]\right\}
\end{aligned}
$$

where $Z(z)$ is the plasma dispersion function and $z=\omega / k_{11}\left(2 \varepsilon T / m_{j}\right)^{l / 2}$. 
Far from mode rational surfaces, where to good approximation $\omega<<k_{11}\left(2 \varepsilon T / m_{j}\right)^{1 / 2}$, the plasma dispersion function can be approximated by the first few terms of a power series: $z(\xi) \approx i \pi^{1 / 2}\left(i-\xi^{2}\right)-2 \xi$. Then for the usual orderings, the untrapped ions provide dispersion and Landau damping:

$$
k^{2} \lambda_{e}^{2} \delta x_{y}(\omega, \underline{k}) \approx \varepsilon \varepsilon_{\frac{\omega}{\omega}}^{1 / 2}{ }^{*}\left[2(1-n) z^{2}+i \pi^{1 / 2}(1-3 n / 2) z^{3}\right],
$$

which for $k_{11} \sim 1 / 2 q R$ and hence $z \sim 2 \omega / \omega_{B i}$ leads to

$$
\begin{aligned}
& \delta n_{U}(\underline{x} ; t) \approx 2 n_{0}\left[-8(1-\eta) \omega_{B i}{ }^{-2}\left(\partial^{2} / \partial t^{2}\right)+8 \pi^{1 / 2}(1-3 \eta / 2) \omega_{B i} i^{-3}\left(\partial^{3} / \partial t^{3}\right)\right] \\
& \left.x(\partial / \partial t) \stackrel{-1}{:}\left(v_{\star}^{\top} \partial / \partial y\right)\right)_{\Phi}(\underline{x} ; t) .
\end{aligned}
$$

The quantity $\delta n(\underline{x}, t)$ is simply given by the sum of the individual contributions, $\delta n(\underline{x} ; t)=\delta n_{B}+\delta n_{T}+\delta n_{j}$. A limit on the magnitude of $\left(\delta n / n_{j}{ }^{\top}\right)$ will be determined as a by-product of the following analysis.

We now return to the augmented Kadomtser-Pogutse fluid equations, Eqs. (1), (3), and (4). We have used the momentum equation (1) to solve for the fluid velocities $\underline{V}_{-}$to third order in $\Phi$. Substituting these solutions for $\underline{v}_{s}$ into the continuity equations (3), expanding Eqs. (3) and (4) also to third order in $\Phi$, and then solving for $n_{e, i}^{\top}$ in terms of an expansion in $\Phi$ will give a nonlinear equation of evolution for $\Phi$. As noted in [15] contributions to $\nabla \cdot\left(n_{s} \underline{V}_{s}\right)$ in Eq. (3) from the diamagnetic drift vanish to all orders in $\dot{\Phi}:$ $\underline{\nabla} \cdot\left[n_{s}\left(c B^{-1} \hat{z} \times \underline{\nabla} p_{s} / n_{s} e_{s}\right)\right]=c \underline{\nabla} \cdot\left(\hat{z} \times \nabla p_{s}\right) / B e_{s} \equiv 0$. We also determined in [15] that $\mid \underline{\nabla} \cdot\left(n_{s}(0)_{V_{s}}(2)|\sim \mathcal{O}| n_{s}^{(0)} k_{k}^{2} v_{s}^{(1) 2} / \Omega_{s}|\ll| \underline{\nabla} \cdot\left(n_{s}^{(1)} \underline{V}_{s}^{(1)}\right)|\sim \mathcal{O}| n_{s}^{(0)} \Phi V(1) / r_{n} \mid\right.$ for parameters typical of large tokamaks. 
We find that at third order in $\Phi$

$$
\left|\underline{\nabla} \cdot\left(n_{s}^{(1)} \underline{V}_{s}^{(2)}\right) / \underline{\nabla} \cdot\left(n_{s}^{(2)} \underline{V}_{s}^{(1)}\right)\right| \sim \theta\left(k_{x}^{2} r_{n}^{2} k_{y} V_{*}^{\top} / \Omega_{s}\right)<<1
$$

and

$$
\underline{\nabla} \cdot\left(n_{s}^{(0)} V_{-s}^{(3)}\right) / \nabla \cdot\left(n_{s}^{(2)} V_{-s}^{(1)}\right) \sim \mathcal{O}\left(k_{x}^{3} r_{n}^{3} k_{y}^{2} v_{k}^{2} / \Omega_{s}^{2}\right)<<1 . \quad \text { Therefore }
$$

$\underline{v}_{e, i}^{\top} \approx C B^{4]} \hat{z X} \underline{\nabla} \phi$ for purposes of expanding the continuity Eq. (3) to $\mathcal{O}_{\Phi}{ }^{3}$ ), which become

$$
\left[(\partial / \partial t)+c B^{-1}\left(\partial_{x} \phi \partial_{y}-\partial_{y} \phi \partial_{x}\right)\right] n_{e, i}^{\top}=-v,+\left[n_{e, i}^{\top}-\varepsilon{ }^{1 / 2} n_{0} \exp ( \pm \Phi)\right] .
$$

From the quasineutrality condition Eq. (4) we determine that

$$
n_{i}^{\top}-n_{e}^{\top}=-g n+2 n_{0}\left(7-\varepsilon^{1 / 2}\right)\left[\Phi+\Phi^{3} / 6+\theta\left(\Phi^{5}\right)\right]
$$

We subtract Eqs. (10)from one another to obtain an expression for $D\left(n_{j}^{\top}-n_{e}^{\dot{T}}\right) / D t$, where $D / D . t \equiv(\partial / \partial t)+C B^{-1}\left(\partial_{x} \phi \partial_{y}-\partial_{y} \phi \partial_{x}\right)$, and use Eq. (11) and the frequency orderings to calculate $n_{e}^{\top}$ to $O\left(\Phi^{3}\right)$ :

$$
\begin{gathered}
n_{e}^{\top} \approx \varepsilon^{1 / 2} n_{0}\left(1+\Phi+\Phi^{2} / 2+\Phi^{3} / 6\right)+\nu_{-}^{-1}(\Phi / D t)\left[2 n_{0}\left(1-\varepsilon^{1 / 2}\right)\left(\Phi+\Phi^{3} / 6\right)-\delta n\right] \\
\quad+\left(\nu_{+} / \nu_{-\infty}\right)\left[2 n_{0}\left(\Phi+\Phi^{3} / 6\right)-\delta n\right] .
\end{gathered}
$$

We substitute this result into Eq. (3) for $n_{e}^{\top}$ to obtain

$$
\begin{gathered}
\nu_{-}^{-1}(D / D t)^{2}\left[2 n_{0}\left(1-\varepsilon^{1 / 2}\right)\left(\Phi+\Phi^{3} / 6\right)-\delta n\right]+(D / D t)\left\{2 n_{0}\left(1-\varepsilon^{1 / 2}\right)\left(\Phi+\Phi^{3} / 6\right)\right. \\
\left.-\delta n+\varepsilon^{1 / 2} n_{0}\left(1+\Phi+\Phi^{2} / 2+\Phi^{3} / 6\right)+\left(\nu_{+} / \nu_{-}\right)\left[2 n_{0}\left(\Phi+\Phi^{3} / 6\right)-\delta n\right]\right\} \\
+v_{+}\left[2 n_{0}\left(\Phi+\Phi^{3} / 6\right)-\delta n\right]=0 \text {. With the use of } D / D t=(\partial / \partial t)
\end{gathered}
$$

$+2 \varepsilon^{-1 / 2} v_{\star}^{\top} r_{n}\left(\partial_{x} \Phi \partial_{y}-\partial_{y} \Phi \partial_{x}-n \Phi \partial_{y}\right), \varepsilon \ll 1$, and the standard frequency order- 
ings, we reduce this expression to

$$
\begin{gathered}
{\left[(\partial / \partial t)+V_{\star}^{\top}(\partial / \partial y)+\nu_{-}^{-1}\left(\partial^{2} / \partial t^{2}\right)-\left(2 n_{0} \Phi\right)^{-1}(\partial \delta n / \partial t)+\nu_{+}\right]\left(\Phi+\Phi^{3} / 6\right)} \\
+(1-\eta) \varepsilon^{-1 / 2} V_{\star}^{\top}(\partial / \partial y) \Phi^{2}+2 \varepsilon^{-1 / 2}\left(\omega_{0} / \nu_{-}\right) V_{\star}^{\top} r_{n} r\left(\partial_{y} \Phi \partial x y-\partial_{x}^{\Phi \partial} y y^{\Phi}\right) \\
\quad: \\
-2 n V_{\star}^{\top}(\partial / \partial y) \Phi^{3} / 6+2 \varepsilon^{-1}(1-n)\left(\omega_{0} / \nu_{-}\right) V_{\star}^{\top} r_{n} r\left(\partial_{x} \Phi \partial_{y}-\partial_{y} \Phi \partial_{x}\right) \partial_{y} \Phi^{2} \approx 0
\end{gathered}
$$

where $\omega_{0} \equiv v_{\star}^{\top} / r$. This is our fundamental nonlinear equation describing the evolution of the dissipative trapped-ion mode in the presence of wave steepening effects.

The validity of Eq. (13) depends upon many approximations, the most important of which are $\varepsilon \ll 1, \Phi \ll 1, \nu_{+} \ll<\omega<\nu_{-}, \omega_{B i} \ll \omega_{B e}$, and $\omega \ll \Omega_{e}, i$. The dependence of the characteristic frequencies and other crucial parameters which appear here on specific tokamak plasma parameters and profiles has been considered in some detail in [15]. The neglect here of electrostatic detrapping $[9,13,19]$ "determines a more restrictive condition than $e_{\phi} / T<1$, namely

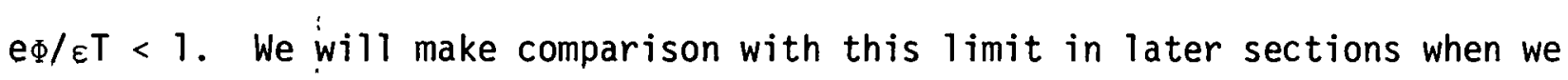
consider approximate steady-state solutions of Eq. (13). In deriving the nonlinear terms in Eq. (13) we have made use of the lowest order linear dispersion relation, $(\partial / \partial t) \approx-V_{\star}^{\top}(\partial / \partial y)$. The validity of this approximation and the accurate correspondence of the linear dispersion relation as determined by our augmented fluid theory with that as derived by a rigorous kinetic theory $[4,5,6]$ set a limit on the size $\delta n / 2 n_{0} \equiv(\partial \delta n / \partial t) / 2 n_{0}, v i z$.

$$
\left|\delta n / 2 n_{0}\right| \ll\left|V_{\star}^{\top}(\partial / \partial y) \Phi\right|
$$

or equivalently

$$
\left|\delta x_{B}(\omega, \underline{k})+\delta x_{T}(\omega, \underline{k})+\delta x_{U}(\omega, \underline{k})\right| \ll\left(k \lambda_{e}\right)^{-2}\left|\varepsilon \varepsilon_{\omega_{e}}^{1 / 2{ }^{*}} /\left(\omega+i v_{+}\right)\right| \approx 2 .
$$


In other words, the rate of Landau damping and the size of the linear frequency shifts due to dispersive effects provided by ion kinetics must be small compared to $\operatorname{Re} \omega \approx \dot{k}_{y} v_{*}^{\top}$.

The remaining sections of this paper consider approximate solutions of Eq. (13). Time dependent and steady-state solutions are constructed, and the stability of nonlinear equilibria is examined where possible. A common feature of all the equilibria found is that nonlinearity, whether due to three-wave or four-wave interactions, creates a balance between a linear source term $\nu_{-}^{-1}\left(\partial^{2} / \partial t^{2}\right)$ and linear dissipative terms arising from $\delta \dot{n} /\left(2 n_{0} \Phi\right)$ and $\nu_{+}$for $\eta<2 / 3$. As the plasma approaches steady-state tine energy flow from the electrons to unstable modes via electron collisions establishes a dynamic equilibrium.with loss processes via mode coupling of unstable to stable modes. Ultimately energy flows to the ions via Landau damping and ion collisions [Fig. 2]: Consideration of (non)conservation laws derived from Eq. (13) by calculating, for example, $\mathrm{d}<\Phi^{2}>/ \mathrm{dt}$ formally demonstrates the different effects produced by the linear driving and damping forces and the nonlinear coupling.

\section{Equilibria Established by Three-Wave Interactions}

\subsection{Radial Instability of One Dimensional Equilibria}

This section considers the nonlinear dynamics of the trappedion modes when the conditions for effective three-wave interactions prevail. Three-wave interactions will occur if the frequency mismatch $\Delta \omega$, given by $\Delta \omega=\omega_{3}-\omega_{1}-\omega_{2}$ is less than the characteristic rate for the three-wave process. Here the frequenctes $\omega_{\ell}, \ell=1,2,3$, satisfy the linear dispersion relation, $\hat{\varepsilon}\left(\omega_{\ell}, k_{\ell}\right)=0$; and the corresponding wave numbers satisfy the condition that $\underline{k}_{3}=\underline{k}_{1}+\underline{k}_{2}$. Refs. 10 and 15 examine this limit and consider the one-dimensional case in which radial vartations are ignored. In the limit 
that three-wave interactions are effective, the $\boldsymbol{\theta}\left(\Phi^{3}\right)$ terms can be discarded. Far from mode rational surfaces $\left(k_{11} \approx 1 / 2 q R\right)$ Eq. (13) can then be written in nondimensional, units as

$$
\begin{aligned}
\frac{\partial \psi}{\partial \tau}+\frac{\partial^{2} \psi}{\partial \xi^{2}}+\alpha \frac{\partial^{4} \psi}{\partial \xi^{4}}+\nu \psi & +\delta_{B} \frac{\partial}{\partial \xi}\left(\frac{\partial^{2}}{\partial \rho^{2}}+\frac{\partial^{2}}{\partial \xi^{2}}\right) \psi+\delta U \frac{\partial^{3}}{\partial \xi^{3}} \psi+\frac{\partial \psi^{2}}{\partial \xi} \\
& +B\left(\frac{\partial \psi}{\partial \xi} \frac{\partial^{2} \psi}{\partial \rho \partial \xi}-\frac{\partial \psi}{\partial \rho} \frac{\partial^{2} \psi}{\partial \xi^{2}}\right) \approx 0,
\end{aligned}
$$

where $\tau \equiv \omega_{u}^{2} t / \nu_{-}, \xi \equiv\left(y-v_{\star}^{\top} t\right) / r, \rho \equiv x / r, \psi \equiv \varepsilon^{1 / 2}(1-n)\left(\nu_{-} / \omega_{0}\right) \Phi$,

$\alpha \equiv \sigma(40)(1-3 n / 2)\left(\nu_{-} / \omega_{0}\right)\left(\omega_{0} / \omega_{B j}\right)^{3}, \nu \equiv \nu_{+} \nu_{-} / \omega_{0}{ }^{2}, \delta_{B} \equiv\left(\nu_{-} / \omega_{0}\right)\left(\rho_{j}{ }^{2} q^{2} / \varepsilon r^{2}\right)$,

$$
\delta_{U} \equiv 8(1-n)\left(\nu_{-} / \omega_{0}\right)\left(\omega_{0} / \omega_{B i}\right)^{2} \text {, and } \beta \equiv 2(1-n)^{-1}\left(\omega_{0} / \nu\right)\left(r_{n} / r\right) \text {. }
$$

A similar equation, but without dispersion $\left(\delta_{B}=\delta_{U}=0\right)$, was the subject of a preliminary investigation by Lovelace and Tang [14].

In Ref. 15 the stability and accessibility of one-dimensional equilibria were studied using Eq. (15) with $(\partial / \partial x) \propto(\partial / \partial \rho) \equiv 0$. In particular, attention was focused on a class of "two-mode" equilibria in which an unstable mode with poloidal mode number $L$ couples to its:stable harmonics $2 L, 3 L, \ldots$ These equilibria were found to be stable to perturbations in the same direction as the wave propagation for $0.6 \leqslant \alpha \mathrm{L}^{2} \leqslant 0.7$. In this section we find that the inclusion of an $x$-dependent variation in the perturbation of the one-dimensional equilibria is always destabilizing for a sufficiently large values of $k_{x} r L b$.

It is useful to define the linear operator $W$, which is simply related to the local growth rate and linear osciliation frequency,

$$
W \equiv-v_{\star}^{\top}\left(\partial / \partial_{y}\right)-v_{-}^{-1}\left(\partial^{2} / \partial t^{2}\right)+\left(2 n_{0} \Phi\right)^{-1}(\partial \delta n / \partial t)-v_{+},
$$

whose Fourier transform in $y$ and Laplace transform in $t$ gives 


$$
\widetilde{w}\left(\omega, k_{y}, i \partial / \partial x ; x\right) \equiv-i k_{y} v_{\star}^{\top}+\omega^{2} / \nu_{-}-v_{+}-i \omega\left(\delta x_{B}+\delta x_{T}+\delta x_{U}\right) / 2
$$

Far from mode rational surfaces, the local value of $\widetilde{W}$ with $\partial / \partial x \equiv 0$ is given simply by $\tilde{W}=-i m \omega_{0}\left[1-\left(\omega_{0} / \nu_{-}\right) \delta m^{2}\right]+\left(\omega_{0}{ }^{2} / \nu_{-}\right)\left(m^{2}-\alpha m^{4}-\nu\right)$, where to lowest order $\omega / \omega_{0} \approx m \equiv k_{y} r$ and $\delta \equiv \delta_{B}+\delta_{U}$. In Ref. 15 the authors found onedimensional equilibria $\psi_{0} \equiv \sum_{n} a_{n L} \exp [i n L(\xi-u \tau)]+c . c$. with amplitudes and group velocity u given by

$$
\begin{aligned}
& a_{2 L}=-\left(\delta L^{2}+u\right) / 2+i \gamma_{L} / 2 L \\
& \left|a_{L}\right|^{2}=-\gamma_{2 L^{\prime}}{ }^{-1}\left(\delta L^{2}+u\right)^{2} / 4-\gamma_{2 L} \gamma_{L} / 4 L^{2} \quad\left[a_{L}=-a_{L}^{\star}\right]
\end{aligned}
$$

and

$$
u=-\delta L^{2}\left(8 \gamma_{L}+\gamma_{2 L}\right) /\left(\gamma_{2 L}+2 \gamma_{L}\right),
$$

where $\gamma_{m} \equiv m^{2}\left(1-\alpha m^{2}\right)-v=\left(\nu_{-} / \omega_{0}^{2}\right)$ Re W.

Finite dispersion can give rise to drifting or convecting steady states with larger saturated amplitudes than for nondispersive two-mode equilibria which are stationary $(u=\delta=0)$ in the electron diamagnetic drift frame [15]. For sufficient dispersion the saturated amplitudes are large enough to make suspect the neglect of the four-wave processes. The dispersion produces a frequency mismatch $\Delta \omega$ whose phase-mixing influence over the time scale of the harmonic generation, viz. $\left(2 \gamma_{L}\right)^{-1}$, becomes sufficient to cancel the energy transfer from the unstable mode to stable modes. Ref. 15 concluded that threewave interactions would not occur for this reason if $\delta>>\alpha 1 / 2$, and made this condition quantitative for the case of ion banana-width dominated dispersion. However, for typical large tokamak parameters and profiles, there is a substantial fraction of the plasma cross-section for which $\delta_{B} / \alpha^{1 / 2}<1$ [15].

For the case where linear dispersion is provided dominantly by untrapped ions, one finds that $\delta_{U} / \alpha{ }^{1 / 2} \sim\left(\nu_{-} / \omega_{B i}\right)^{1 / 2}$. In order to guarantee the validity 
of our model equations the inequalities (14) must be satisfied. This together with the facts that $v_{+} \sim\left(m_{e} / m_{j}\right)^{1 / 2} v_{-} / 2$ and for instability $1 / 4 \alpha>v$ tends to restrict parameter choices to $\nu_{-} / \omega_{B i} \sim \mathcal{E}(1)[1 b]$. Hence our model is valid only in a small region of parameter space for which the effectiveness of threewave coupling is probably marginal due to the relatively significant degree of dispersion $\delta_{U}$. As we approach linear marginal stability $1 / 4 \alpha-v \rightarrow 0$, any dispersion whatsoever will render three-wave coupling ineffective since the frequency mismatch $\Delta \omega \propto \delta$ will greatly exceed $2 \gamma_{L} \rightarrow 0$, the characteristic rate of the harmonic generation. This motivates the detailed consideration of the $\boldsymbol{\theta}\left(\Phi^{3}\right)$ terms in Eq. (13), which is discussed in Sec. 4.

We point out that in both Refs. 10 and 15 appears the unfortunate use of the terminology "near marginal stabjlity" to describe plasma parameter choices for which $\alpha$ is not too small i.e. $10^{-2} \leq \alpha<1$, which is consistent with $v_{-} / \omega_{\mathrm{Bi}} \sim \boldsymbol{O}(1)$ and $\nu_{+} \ll \omega_{0} \ll v_{-}$. The intent was to indicate that for finite rather than infinitesimal $\alpha$ there are not too many unstable modes $\left(\sim_{\alpha}^{-1 / 2}\right)$ and that the maximum growth rate is not too large $\left(\sim \omega_{0}^{2} / 4 \alpha \nu_{-}\right)$. However, it was assumed in both [10] and [15] that the maximum growth rate was nevertheless well above threshold: Im $\omega \propto(1 / 4 \alpha-v) \sim 1 / 4 \alpha \gg v$. A stricter definition of linear marginal stability is that the linear growth rate of the most unstable mode approaches zero (threshold), $1 / 4 \alpha=O(v)$, and is the definition we use throughout the present discussion.

To demonstrate instability of the one-dimensional equilibria subject to perturbations with $x$-dependent variation, it is sufficient to consider the simplest case of no dispersion. The equilibrium $\psi_{0}$ satisfies the equation

$$
\left(\frac{\partial^{2}}{\partial \xi^{2}}+\alpha \frac{\partial^{4}}{\partial \xi^{4}}+\nu\right) \psi_{0}+\frac{\partial \psi_{0}^{2}}{\partial \xi}=0,
$$

with amplitudes $a_{n L}, n=1,2$ given by Eq. (17) in the $\delta=0$ limit. The per- 
turbation $\delta \psi=\exp (-i \Omega \tau) \exp \left(i k_{x} r \rho\right) \sum_{n-} a_{m+n} L^{-} \exp [i(m+n-L) \xi]+c . c$. satisfies the linearized equation

$\left(\frac{\partial}{\partial \tau}+\frac{\partial^{2}}{\partial \xi^{2}}+\alpha \frac{\partial^{4}}{\partial \xi^{4}}+\nu\right) \delta \psi+2 \frac{\partial}{\partial \xi}\left(\psi_{0} \delta \psi\right)+\beta\left(\frac{\partial}{\partial \xi} \psi_{0} \frac{\partial^{2} \partial \psi}{\partial \rho \partial \xi}-\frac{\partial \delta \psi}{\partial \rho} \frac{\partial^{2} \psi_{0}}{\partial \xi^{2}}\right)=0$.

We Laplace transform this expression $\frac{\partial}{\partial \tau} \rightarrow-i \Omega$, where $|\Omega| \ll\left(\nu_{-} / \omega_{0}\right)|m+n-L|$, and obtain

$$
\begin{aligned}
& \qquad\left[-i \Omega-\left(m+n L+n^{-} L\right)^{2}+\alpha\left(m+n L+n^{-L}\right)^{4}+v\right] \delta a_{m+\left(n+n^{\prime}\right) L}= \\
& -i \sum_{n, n^{\prime}} 2\left[m+\left(n+n^{\prime}\right) L\right]+k_{x} r_{\beta}\left[(n L)^{2}-n L\left(m+n^{\prime} L\right)\right] \quad a_{n L} \delta a_{m+n^{\prime} L}, \\
& \text { where } n= \pm 1, \pm 2, \quad n^{\prime}=0, \pm 1, \pm 2, \ldots \text { and } a_{-n L}=a_{n L}^{\star} .
\end{aligned}
$$

Eq. (19) constitutes an infinite matrix equation. Following the example of Ref. 15, we use the fact that modes with large poloidal mode number suffer severe Landau damping and contribute negligibly to the perturbation eigenfunctions. This justifies truncation of the set of perturbed Fourier modes to only the $m, m \pm L, m \pm 2 L$ modes. The stability problem is therefore reduced to the calculation of the eigenvalues of a $5 \times 5$ matrix whose elements are given by Eq. (19). Figs. (3a), (3b), and (3c) display the results for $\Omega / l^{2}$ as function of $k_{x} r L_{\beta}$ with parameter choices $L=8, m=1$, and $\alpha L^{2}=.56, .64$, and .72 respectively. For $k_{x} \mathrm{rL}_{\beta} \rightarrow 0$ we recover exactly the quantitative results of the one dimensional analysis in [15] and find that the two-mode equilibria are stable to perturbations with $y$ variation for $0.6<\alpha L^{2}<0.7$. For $\left|k_{x} r L_{B}\right|>0.1$ there is instability. Direct numerical integration of Eq. (15) has quantitatively confirmed these results. The numerical integrations employed the technique described in [15] with the addition of an $x$ grid and difference operator $\partial f / d x \rightarrow[f(x+\Delta x)-f(x-\Delta x)] / 2 \Delta x$. The instability of the one-dimensional 
two-mode equilibria to perturbations with transverse (radial) variation motivates us to investigate the nonlocal, fully two-dimensional mode structure of the trapped-ion instability.

\subsection{Two Dimensional Equilibria Established by Three-wave Interactions}

Linear calculations of the radial mode structure have shown that, of the many effects arising from the radial variation of the plasma parameters, current, and magnetic field, magnetic shear can have the most pronounced influence $[6,177$. Untrapped ions can provịde ștrọnq Làndau damping if $\omega / k_{11} \sim\left(T_{j} / m_{j}\right)^{1 / 2}$. Since effectively $0 \leq k_{11} \approx|\dot{\ell} q(r)-m| / q(r) R \leq 1 / 2 q R$, theire is a strong variation of the Landau damping of the trapped-ion mode with respect to the radial separation from the mode rational surface where $k_{11}(r)=0$. The circulating ions respond adiabatically, $\omega / k_{11} \ll\left(T_{j} / m_{j}\right)^{l / 2}$, where $k_{11} \approx 1 / 2 q R$ very far from the mode rational surface. There is severe Landau damping in a layer fairly close to the rational surface, and then again weak damping for $\omega / k_{11} \gg\left(T_{i} / m_{i}\right)^{1 / 2}$ at the rational. surface, where the circulating ions respond hydrodynamically. For monotonic q profiles the separation between rational surfaces is given approximately by $\Delta r_{s} \equiv 1 / x q \approx r / m$. Hence, rur muderately large poloidal quantum number $m$, the characteristic length over which the untrapped ion response $\delta x_{U}$ varies dramatically is much shorter than any of the characteristic radial lengths over which other coefficients in Eq. (13) vary. This motivates us to discard all radial variation of coefficients in Eq. (13) except for that arising from $\delta x_{U}(\omega, \underline{k} ; x)$.

Gladd and Ross [6] have found that the strong dependence of $\delta x_{U}$ on radial separation from the rational surface generally produces reflection and strong dissipation in the vicinity of $k_{11}=0$. They also find that otherwise $\delta x_{U}$ is fairly constant in most of the region between rational surfaces. The net 
effect is to cause radial nodes in the waveform fairly close to the mode rational surfaces for a given mode. As a useful theoretical "ansatz" which allows us to make considerable analytic progress in finding relatively simple nonlocal solutions of Eq. (13), we also take $\delta x_{U}$ to be independent of $x$ by setting $k_{11} \approx 1 / 2 q R$ but require the spontaneously excited unstable modes to have vanishing amplitude at their mode rational surfaces.

We will construct steady-state solutions of

$$
\frac{\partial}{\partial \tau} \psi-\bar{W} \psi+\frac{\partial}{\partial \xi} \psi^{2}+\beta\left(\frac{\partial}{\partial \xi} \psi \frac{\partial^{2} \psi}{\partial \rho \partial \xi}-\frac{\partial \psi}{\partial \rho} \frac{\partial^{2} \psi}{\partial \xi^{2}}\right)=0,
$$

where $\bar{W} \equiv \nu_{-} W / \omega_{0}^{2}[$ see Eq. (16a)]. As before, solutions are considered in which a single linearly unstable mode couples with itself to generate linearly stable modes. The total disturbance $\psi$ will then vanish at the mode rational surfaces of the single unstable mode, $\psi(x=0)=\psi\left(x=\Delta r_{s}\right)=0$, and is periodic in $y$, $\psi(y+2 \pi r)=\psi(y)$.

We expand the potential in ascending powers of the wave amplitude of the unstable mode: $\psi=\psi^{(1)}+\psi^{(2)}+\ldots$ where $\psi^{(1)}=\sin \left(k_{\rho}\right)\left[a_{11} \exp (i L \xi)+c . c.\right]$, ${ }_{\psi}^{(?)}=\sum_{n, n^{\prime}} a_{n n^{\prime}} \sin \left(n^{\prime} k \rho\right) \exp (i n L \xi)+c . c$. , and $k \equiv \pi r / \Delta r_{s} \approx \pi L$. In general, there can be a poloidal drift at steady state, $\partial / \partial \tau \rightarrow-u \partial / \partial \xi$. To second order in $\left|a_{11}\right|$ we obtain

$$
[-u(\partial / \partial \xi)+\bar{W}] \psi(2) \approx-(\partial / \partial \xi) \psi(1) 2-6\left(\frac{\partial}{\partial \xi} \psi^{(1)} \frac{\partial^{2}}{\partial \xi \partial \rho} \psi(1)-\frac{\partial}{\partial \rho} \psi(1) \frac{\partial^{2}}{\partial \xi^{2}} \psi(1)\right)
$$

and

$$
\begin{aligned}
& {[-u(\partial / \partial \xi)+\bar{W}] \psi(1) \approx<\left[-(\partial / \partial \xi) \lambda_{\psi}(1)_{\psi}(2)-\beta\left(\frac{\partial}{\partial \xi} \psi^{(1)} \frac{\partial^{2}}{\partial \xi \partial \rho} \psi(2)+\frac{\partial}{\partial \xi} \psi(2)\right.\right.} \\
& \left.\left.\left.x \frac{\partial^{2} \psi^{(1)}}{\partial \xi \partial \rho}-\frac{\partial \psi^{(1)}}{\partial \rho} \frac{\partial^{2} \psi^{(2)}}{\partial \xi^{2}}-\frac{\partial \psi(2)}{\partial \rho} \frac{\partial^{2} \psi^{(1)}}{\partial \xi^{2}}\right)\right], \psi^{(1)}\right\rangle \frac{\psi^{(1)}}{\left\langle\psi(1), \psi^{(1)}\right\rangle},
\end{aligned}
$$

where we define an inner product $\left\langle f, g>\equiv\left(2 \pi \Delta r_{s} / r\right)^{+1} \int_{0}^{2 \pi} d \xi \int_{0}^{\Delta r_{s} / r} d \rho f g\right.$. 
The orthogonality of $\sin (\ell x)$ and $\sin (m x), \ell \neq m$, on the interval $[0, \pi]$ can then be used to Fourier expand the r.h.s. of Eq. (21a) and determine the Fourier coefficients $a_{n n}$, for $\psi^{(2)}$. We find that

$$
\begin{aligned}
& a_{02}=\left.k L^{2}{ }_{B} a_{11}\right|^{2} \tilde{W}_{02}^{-1} \\
& a_{21}=(16 / 3 \pi) i L a_{11}{ }^{2}\left(\tilde{W}_{21}-i 2 L u\right)^{-1} \\
& a_{23}=-(16 / 15 \pi) i L_{i j}{ }^{2}\left(w_{23^{-}}-i ? 111\right)^{-1}
\end{aligned}
$$

where $\tilde{W}_{n n^{-}} \equiv \tilde{W}\left(\omega, k_{y}=n L r^{-1}, k_{x}=n^{-} \pi \Delta r_{s}^{-1}\right)$ as defined in Eq. (16b).

As noted earlier the formation of an equilibrium depends on the nonlinear stabilization of the linearly unstable mode by means of mode coupling. To justify truncation of $\psi$ at $\theta\left(\left|a_{11}\right|^{2}\right)$ all the Fourier modes in Eq. (22a) must be linearly damped, $\operatorname{ReW}_{m n}<0$. Furthermore, the subsequent couplings of ${ }_{\psi}{ }^{(1)}$. with $\psi^{(2)}$, etc. generate higher order modes which must also be linearly stable. Examination of the linear mode structure determined by Eq. (16) allows the identification of the relevant energy sinks. At $k_{y}=0$ the modes are damped by ion collisions. At very short poloidal wavelengths, $m \equiv k_{y} r>\alpha^{-1 / 2}$, the modes are linearly stabilized by ion Landau damping provided $\eta_{j}<2 / 3$. For increasing effective radial wavenumber $k_{x} r$, the modes are stabilized in a monotonically increasing fashion [6]. This effect arises as a consequence of the finite ion banana-width reduction of the mode frequency $\propto k_{x}^{2} \rho_{i}^{2} q^{2} \varepsilon^{-1}$ and $i$ ts subsequent influence on the growth rate, which involves the frequency dependent electron driving term and the ion Landau damping terms. 
To provide for the lowest order finite ion banana-width effects $\left(k_{x}^{2} \rho_{j}^{2} q^{2} \varepsilon^{-1}<1\right)$ in the linear dispersion relation, we perturbatively include a frequency correction in evaluating the $\omega$-dependent terms in $\tilde{W}$, i.e. $\omega \approx k_{y} v_{*}^{\top}\left(1-k_{x}^{2} b_{i}^{2}-k_{y}^{2} b_{i}^{2}\right)$ where $b_{i}{ }^{2} \equiv \rho_{i}{ }^{2} q^{2} / \varepsilon$ in evaluating $\omega^{2} / \nu_{-}, \delta x_{T}$, and $\delta x_{U}$. For simplicity in constructing an equilibrium, we hypothesize plasma parameters such that the Fourier mode with $k_{y} r \approx(2 \alpha)^{-1 / 2}$ and $k_{x} r=\pi r / \Delta r_{s}=$ $\pi k_{y} r$ is unstable, but the mode with $k_{x} r=2 \pi r / \Delta r_{s}$ and the same $k_{y}$ is stable (Fig. 4). The studies of Eq. (15) appearing in [14] did not have an energy sink at short effective radial wavelengths. Therefore the waveforms could be expected to undergo continued fluid steepening in the radial $(x)$ direction without saturating.

Reduction of Eqs. (22a) and (22b) can be made by substitution of the amplitudes determined in (22a) into (22b):

$$
\begin{aligned}
\left(\tilde{w}_{11^{-}} i L u\right)= & -(16 L / 3 \pi)^{2}\left|a_{11}\right|^{2}\left(\tilde{w}_{2 T^{-}}-i 2 L u\right)^{-1}-(16 L / 15 \pi)^{2}\left|a_{11}\right|^{2}\left(\tilde{w}_{23}-i 2 L u\right)^{-1} \\
& -2 k^{2} L^{4} B^{2}\left|a_{11}\right|^{2} \tilde{w}_{02}^{-1}+\ldots
\end{aligned}
$$

which gives the approximate expression

$$
\left|a_{11}\right|^{2} \approx-\left(\tilde{W}_{11^{-}}-i L u\right)\left[(16 L / 3 \pi)^{2}\left(\tilde{W}_{21}-i 2 L u\right)^{-1}+2 k^{2} L^{4} \beta^{2} \tilde{W}_{02}^{-1}\right]^{-1}
$$

upon truncation of smaller terms.

The real and imaginary parts of Eq. (23) determine two equations for $\mid a_{11} j^{2}$ and $u$,whose simultaneous solution gives

$$
u^{3}+a u^{2}+b u+c=0,
$$

$$
\begin{aligned}
& \text { where } a \equiv-\left(I_{11}+I_{21}\right) L^{-1}, b \equiv\left[I_{21}\left(4 I_{11}+I_{21}\right)+R_{21}^{2}-\left(2 R_{11}+R_{21}\right) d^{-1}\right]\left(4 L^{2}\right)^{-1}, \\
& C \equiv\left[-I_{11} I_{21}^{2}-R_{21}^{2} I_{11}+\left(R_{11} I_{21}+I_{11} R_{21}\right) d^{-1}\right], d \equiv 2(3 \pi / 16)^{2}(k L \beta)^{2} v^{-1}, R_{m n} \equiv R_{e} \widetilde{W}_{m n},
\end{aligned}
$$


and $I_{m n} \equiv \tilde{I m W}_{m n}$. From the standard discriminant for cubic equations one can determine under what circumstances there are one, two, or three real solutions for $u$. For a given velocity u satisfying Eq. (24), $\left|a_{11}\right|^{2}$ can be determined from either the real or the imaginary. parts of Eq. (23) and must be positive definite for an equilibrium to exist. The real part of Eq. (23) gives

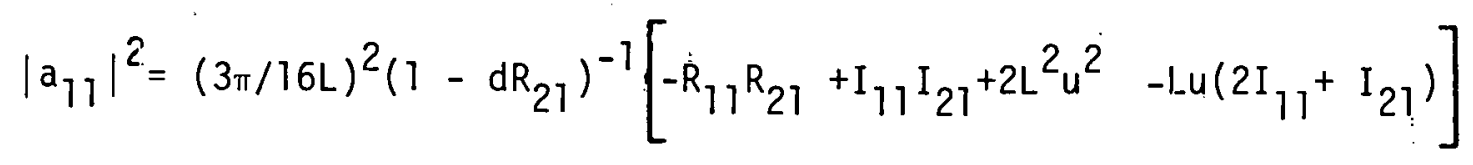

We have used the imaginary part of Eq. (23) to provide an alternative determination of $\left|a_{117}\right|^{2}$ and thus check the consistency of specific numerical evaluations of $u$ and $\left|a_{11}\right|^{2}$.

To make qualitative connection with the one-dimensional two-mode equilibria, we compare Eq. (25) to Eqs. (17). $R_{m n}$ corresponds to a linear growth/ damping rate. If we set $d \propto k_{\beta} \equiv 0$ and ignore dispersion, $I_{m n} \equiv 0$, then $u=0$ and $\left|a_{11}\right|^{2}=-(3 \pi / 8)^{2} R_{11} R_{21} / 4 L^{2}$. This aqrees with Eqs.(17) in the nondispersive limit except for the geometrical factor $(3 \pi / 8)^{2}$ which is due to the reduction in coupling efficiency caused by the $\sin \left(k_{\rho}\right)$ radial mode structure. Fur. typical large tokamak parameters and choice of modes for which $R_{11}<0$ and $R_{21}>0$, dispersive equilibria with single real-valued solution for $u$ as well as nondispersive equilibria with $u=0$ exist. Dispersion tends to increase the saturated mode amplitude, $\mathrm{I}_{11} \mathrm{I}_{21},: 2 \mathrm{~L}^{2} \mathrm{u}^{2}>0$; but this is compensated by the amplitude reduction provided by the increased coupling when $\beta \neq 0$. For typical parameters and a choice of modes such that $R_{21}<0$, then $\left|\underset{d}{d} R_{21}\right|>>1$; and $\left|a_{11}\right|^{2}$ as estimated by Eq. (25) can be significantly smaller than the onedimensional result Eqs. (17).

The mode amplitudes approach steady state in a manner much the same as described for the one-dimensional two-mode configurations [15]. The mode amplitude of the fundamental $\psi^{(1)}$, presumed to be thermally excited at an 
at an initially small amplitude, grows at an exponential rate given by $\operatorname{Re} \tilde{W}$ By beating with itself it generates the damped modes comprising $\psi^{(2)}$ at an exponential rate $2 \operatorname{Re} \tilde{\mathrm{N}}_{11}$ until just before saturation, at which time the growth of both $\psi^{(1)}$ and $\psi(2)$ diminishes rapidly.

We observe that the equilibrium we have approximately constructed in Eqs. (23), (24), and (25) has no explicit evidence of the energy sink at large $k_{x}$. However, implicit in the analysis is a truncation of the Fourier modes at third order and higher in $\left|a_{11}\right|$, which requires that modes at large $k_{x}$ be linearly stable or damped. There has also been a truncation of the Fourier expansion of $\sin ^{2}\left(k_{\rho}\right)$, i.e. $\sin ^{-2}\left(k_{\rho}\right) \approx(8 / 3 \pi) \sin \left(k_{\rho}\right)-(8 / 15 \pi)$ $x \sin \left(3 k_{\rho}\right)$. In fact, because in the calculation of $\left|a_{11}\right|^{2}$ the square of these Fourier coefficients enters, only the first term of the preceding expansion is actually saved in Eq. (23). These approximations are responsible for small errors in the equilibrium which fortunately are not too important for the overall scaling of the saturated amplitude and associated anomalous transport. However, a stability analysis of these equilibria, similar to that described in Sec. 3.1, may be quite sensitive to these errors and give misleading or totally erroneous results.

We have undertaken a linear stability analysis of the equilibrium $\psi_{0}=a_{11} \sin \left(k_{\rho}\right) \exp \left[i L\left(\xi-u_{\tau}\right)\right]+a_{02} \sin \left(2 k_{\rho}\right)+a_{21} \sin \left(k_{\rho}\right) \exp \left[i 2 L\left(\xi-u_{\tau}\right)\right]+$ c.c., where the amplitudes and group velocity $u$ are given by Eqs. (22a), (23), and (24). We find frequency eigenvalues giving instability, which are much larger in magnitude than $\left(\nu_{-} / \omega_{0}\right) \mathrm{L}$. This violates our approximate use of $w \approx k_{y} v_{\star}^{\top}\left(1-k_{x}^{2} b_{i}^{2}-k_{y}^{2} b_{i}^{2}\right)$ in evaluating $\tilde{w}\left(\omega, k_{y}, k_{x}\right)$. Hence, the perturbative approach used here formally breaks down. However, this should not ncessarily diminish the value of our approximate equilibria calculation which may nevertheless give an accurate approximation of the correct scaling of the saturated mode amplitudes and transport. For example, in Ref. 15 it was deter- 
mined that even when the simple one-dimensional two-mode equilibria were unstable, the energy and transport of the numerically obtained asymptotic spectra were bounded and scaled in agreement with the two-mode theory.

The lack of dependence of our approximate equilibrium solutions upon the details of the energy sink at short effective wavelengths in the radial direction, although perhaps troublesome for the stability analysis, is an advantage. in other respects. We are able to deduce the approximate scaling of the saturated amplitude and transport without demanding a precise calculation of the linear radial mode structure and its effects upon the linear mode frequency. Consider the approximate expression for $\operatorname{ReW}(\omega, \underline{k})$ given by the layior series expansion.

$$
\operatorname{Re} \tilde{W}(\omega, \underline{k}) \approx \operatorname{Re}_{0}\left(\omega=k_{y} v_{\star}^{\top}, \underline{k}\right)+\frac{\partial \operatorname{Re} \tilde{W}_{0}\left(\omega=k_{y^{\prime}} v_{\star}^{\top}, \underline{k}\right)}{\partial \omega} \delta(\omega)+\ldots,
$$

where $\tilde{W}_{0}$ is given by the local limit of Eq. (16b) with $i \partial / \partial x \rightarrow k_{x}$ and $\delta \omega \equiv$ $-k_{y} v_{*}^{\top}\left(k_{x}^{2} b_{i}^{2}+k_{y}^{2} b_{i}^{2}\right)$. It is straightforwara to aemonstrate that in the clodel nom1 inear equilibria described by EqS. $(21-25)$, slabilization (ReN $\left.\tilde{N}_{0} \leq 0\right)$ of modes with $k_{y}=L / r$ and $k_{x}=n_{\pi} / \Delta r_{s}, n \geq 2$, requires $k_{x}{ }^{2} b_{i}{ }^{2} \sim \sigma_{(1)}$ ror the case that the single unstable mode $\left(k_{y}=L / r\right.$ arid $\left.k_{x}=\pi / \Delta r_{s} \approx k_{y}\right)$ is well above linear marginal stability and has nearly maximal linear growth rate. The implicit violation (for $n \geq 2$ ) of the conditions that $k_{x}{ }^{2} b_{i}{ }^{2} \ll 1$ and that $|\delta \omega| \ll k_{y} v_{*}^{T}$ indicates the weakness of the weakly perturbative addition of kinetic effects to our fluid model. We point out, however, that these conditions are fairly well satisfied by the modes explicitly retained in these equilibria. The next section describes a calculation appropriate for plasmas very close to marginal stability, in which the assumptions made by our model equations are very well justified. 
4. Two Dimensional Equilibria Near Marginal Stability

4.1 Equilibria Established by Four-wave Insteractions

Near linear marginal stability the electron driving term in

the linear dispersion relation is nearly cancelled by the damping terms produced by ion collisions and ion Landau damping. We specialize our study to the case of a single weakly unstable mode and consider the linear dispersion relation given by Eqs. (16) in the local limit:

$\omega_{L} \equiv \Omega_{L}+i \gamma_{L}=-I m \tilde{W}+i \operatorname{Re} \tilde{W}=k_{y} v_{\star}^{\top}\left[1-k_{x}{ }^{2} b_{i}{ }^{2}-k_{y}{ }^{2} b_{i}{ }^{2}-8(1-n)\left(\omega / \omega_{B i}\right)^{2}\right]$

$$
+i\left[\left(\omega^{2} / \nu_{-}\right)-\nu_{+}-\gamma_{L D}\right]
$$

where $\gamma_{L D}=(1-3 n / 2)\left(\omega / \omega_{B i}\right)^{2} \omega\left[A^{-}\left(\omega / \omega_{B i}\right)+8 \pi^{1 / 2}\left(k_{y} v_{\star}^{\top} / \omega_{B i}\right)\right], A^{-}=\mathscr{O}(20)$, and to good approximation $\omega \approx \mathrm{k}_{y} V_{\star}^{\top}$ on the r.h.s. The effective poloidal wavenumber of the single marginally stable mode is determined by $d \gamma_{L} / d k_{y}=0$ and by a consistency relation between $\alpha$ and $v, \gamma_{L} \approx\left(\omega_{0}^{2} / v_{-}\right)\left(m^{2}-\alpha m^{4}-v\right)=0$, where $m \equiv k_{y} r$. These give $\alpha m^{2}=1 / 2$ and $4 v \alpha=1$. We set $k_{x} \approx \pi k_{y}$ for this mode in the spirit of our earlier arguments and those of Ref. 6 .

The single unstable wave grows at first from some initial value, which is assumed small, at its linear growth rate $\gamma_{L}$. The coupling of the mode with itself through the nonlinear terms in Eq. (13) drives to finite amplitude its harmonics which would otherwise be thermally excited to much smaller amplitudes. Harmonics will be generated at all orders in the amplitude of the fundamental and with growth rates given by the corresponding integral multiples of the growth rate $\gamma_{L}[15]$.

The preceding arguments concerning nonlinear effects tacitly assume that phase matching conditions are satisfied, i.e. the sum or difference of the wavenumbers and corresponding linear normal mode frequencies of the weakly 
coupled modes must nearly equal the wavenumber and frequency of another normal mode. When there is a mismatch $\Delta \omega$ in the frequencies, the effectiveness of the coupling process is reduced; and coupling virtually ceases because of the loss of phase coherence when $\Delta \omega$ greatly exceeds the characteristic rate of the process. Because harmonic generation has a characteristic rate scaling as an integral multiples of $\gamma_{L}$, any finite frequency mismatch will be sufficient to completely inhibit coupling as $\gamma_{L}$ is decreased to zero at marginal stability. For linear normal modes with dispersion relation given by Eq. (27), all threewave interactions have a finite mismatch frequency and are therefore ineffective for systems near marginal stability. Eq. (13) then becomes

$$
\begin{aligned}
& {[(\partial / \partial t)-W]\left(\Phi_{i}+\Phi^{3} / 6\right)-2 n_{i} V_{\star}^{\top}(\partial / \partial y) \Phi^{3} / 6} \\
& \quad+2 \varepsilon^{-1}(1-n)\left(\omega_{0} / \nu_{-}\right) V_{\star}^{\top} r_{n} r \cdot\left(\partial_{x} \Phi \partial_{y}-\partial_{y} \Phi \partial_{x}\right) \partial \Phi{ }^{2} \approx 0 .
\end{aligned}
$$

We ignore parametric decays, [20], whose characteristic growth rates scale typically as a positive power of the wave amplitude of the "pump", the wave undergoing decay into other waves. Since the modes we consider here are assumed to grow from an initially small amplitude, the growth rates for parametric decay are quite small initially. Meanwhile harmonic generation proceeds at an amplitude-independent rate proportional to. $\gamma_{L}$. Furthermore, dissipation of the decay products and frequency mismatch, typically reduce the growth rates for parametric decay, produce an instability threshold condition on the amplitude of the pump wave, and therefore further decrease the likelihood and efficiency of parametric decay. The cubic nonlinearity in Eq. (28) requires us to survey all possible four-wave interactions. Because of the dispersion present in the linear normal mode frequencies, given by Eq. (27), many of the four-wave interactions can be ruled out because of the effects of frequency mismatch. A subclass of four-wave interactions possessing identically zero frequency mismatch 
have the trivial phase-matching conditions .

$$
\begin{aligned}
& \underline{k}_{1}+\underline{k}_{2}=\underline{k}_{1}+\underline{k}_{2} \\
& \Omega_{L}\left(\underline{k}_{1}\right)+\Omega_{L}\left(\underline{k}_{2}\right)=\Omega_{L}\left(\underline{k}_{1}\right)+\Omega_{L}\left(\underline{k}_{2}\right) .
\end{aligned}
$$

We demonstrate in this sub-section that the four-wave interaction of the unstable mode with itself $\left(\underline{k}_{1}=\underline{k}_{2}=\underline{k}\right)$ induces a frequency shift which causes the mode to be nonlinearly stabilized by modification of the linear driving and damping mechanisms. The self-coupling of the unstable mode also induces frequency shifts in the otherwise stable modes, $\underline{k}_{1} \neq \underline{k}_{2}=\underline{k}$. If this frequency shift is large enough to nonlinearly destabilize a linearly damped mode, then the equilibrium established by the self-coupling of the single linearly unstable mode is clearly unstable (Sec. 4.2). For $\underline{k}_{1}, \underline{k}_{2} \neq \underline{k}$ the wavenumber of the single unstable mode, all four waves are linearly damped; and the four-wave process is unimportant unless one of the modes has been nonlinearly excited to finite amplitude by some other process. If this should occur the coupling described in Eq. (29) again produces a frequency shift.

To consider the self-coupling of the single unstable mode we describe its amplitude by

$$
\Phi(x, y ; t) \equiv \Phi_{0} \sin \left(k_{x} x\right) \exp \left[i k_{y}-i \int_{0}^{t} d t^{\prime} \omega\left(t^{-}\right)\right]+c . c .,
$$

where in general $\omega(t) \equiv \omega_{L}+\delta \omega(t) \equiv \Omega_{L}+\delta \Omega(t)+i \gamma_{L}+i \delta \gamma(t)$ and $\omega_{L}=\Omega_{L}+i \gamma_{L}$ is given by Eq. (27) In the absence of nonlinear effects, $\omega(t)=\omega_{L}$ in order that $\Phi$ satisfy Eq. (28). Hence, $\delta \omega(t)$ is due to nonlinear coupling and is very small if $\Phi$ is very weakly nonlinear, i.e. $\left|\delta \omega / \omega_{L}\right| \propto \boldsymbol{\theta}\left(\Phi^{2}\right) \ll 1$. 
The nonlinear terms arising from $[(\partial / \partial t)-W] \Phi^{3} / 6$ are much smaller by $\theta\left(\gamma_{L} / k_{y} V_{\star}^{\top}\right)$ than the other nonlinear terms in Eq. (28) and are therefore neglected consistent with earlier approximations. The nonlinear term in Eq. (28) containing $\left(\partial_{x} \Phi \partial_{y}-\partial_{y} \Phi \partial_{x}\right) \partial_{y}{ }^{2}$ generates modes with $x$-dependent structure given by $\sin ^{2}\left(k_{x} x\right) \quad \cos \left(k_{x} x\right)=(1 / 2) \sin \left(k_{x} x\right) \sin \left(2 k_{x} x\right)=(1 / 4)\left[\cos \left(k_{x} x\right)\right.$

$\left.-\cos \left(3 k_{x} x\right)\right]=\sum_{n=1}^{\infty} a_{2 n} \sin \left(2 n k_{x} x\right)$, and hence does not contribute to the selfcoupling because no mode with variation $\sin \left(k_{x} x\right)$ appears in the series. The remaining nonlinear term, which contains $(\partial / \partial y)_{\Phi}^{3} / 6$, generates modes with structure $\sin ^{3}\left(k_{x} x\right)=(3 / 4) \sin \left(k_{x} x\right)-(1 / 4) \sin \left(3 k_{x} x\right)$, and therefore provides for self-coupling. Therefore, from Eq. (28) we obtain

$-i_{\omega_{L}}-i \delta \omega(t)-\tilde{w}\left(\omega_{L}+\delta \omega, \underline{k}\right)-\left.i(3 / 4) n k_{y} V_{\star}^{\top}\right|_{0} \Phi_{0}^{2} \exp \int_{0}^{t} d t^{\prime} 2 \operatorname{Im} \omega\left(t^{\prime}\right)=0$.

We Taylor-series expand $\tilde{W}(\omega, \underline{k})$ around $\tilde{W}\left(\omega_{L}, \underline{k}\right)=i_{\omega_{L}}$ in the small variable $\delta \omega / \omega_{L} \propto O\left(\left.T \pi\right|^{2}:\right.$

$$
\begin{aligned}
-i \delta \omega(t)-\left.\frac{\partial}{\partial \omega} \tilde{W}\right|_{(j)} \delta \omega & -\left.\frac{\partial^{2}}{\partial \omega^{2}} \tilde{W}\right|_{\omega_{L}} \frac{\delta \omega^{2}}{2}+\ldots \\
& -i(3 / 4)_{\eta} i k_{y} V_{*}^{\top}\left|\dot{\Phi}_{0}\right|^{2} \exp \left[\int_{0}^{t} d t 2 \operatorname{Im} \omega\left(t^{\prime}\right)\right] \approx 0 .
\end{aligned}
$$

Saturation can occur if the net growth rate can be nonlinearly. reduced to zero, $\operatorname{Im}_{\omega}(t)=\gamma_{L}+\delta \gamma(t) \rightarrow 0$. Then at saturation $\delta \gamma(\infty)=-\gamma_{L}$. From the rea 1 : and imaginary parts of Eq. (32) we obtain to lowest order in $\left|\delta \omega / \omega_{L}\right| \cdot$ and $\left|k_{y} V_{\star}^{\top} / \nu_{-}\right|$

$$
\delta \Omega(\infty) \approx-(3 / 4) \eta k_{y} V_{\star}^{\top}\left|\Phi_{\infty}\right|^{2}
$$

and

$$
-\gamma_{L}=\left.\delta \gamma(\infty) \approx \frac{\partial}{\partial \omega} \operatorname{Re} \tilde{W}\right|_{\omega_{L}} \delta \Omega(\infty)+\left.\frac{\partial^{2}}{\partial \omega^{2}} \operatorname{Re} \tilde{W}\right|_{\omega_{L}} \frac{\delta \Omega}{2}(\infty)^{2}
$$


where $\left|\Phi_{\infty}\right|^{2} \equiv\left|\Phi_{0}\right|^{2} \exp \left[\int_{0}^{\infty} d t^{-}-2 \operatorname{Im} \omega\left(t^{\prime}\right)\right]$.

Evaluation of $(\partial / \partial \omega) \operatorname{Re} \tilde{W}$ and $\left(\partial^{2} / \partial \omega^{2}\right)$ ReN completes the solutions of $\delta \Omega(\infty)$ and $\left|\Phi_{\infty}\right|$ and can be used to justify the approximation that $|\delta \gamma| \ll|\delta \Omega|$ which has been used in deriving Eqs. (33). For the most unstable mode, $\alpha m^{2}=1 / 2$, we deduce from Eq. (27) that $(\partial / \partial \omega) \operatorname{Re} \tilde{W} \approx m_{\omega_{0}} / 4 \nu_{-}$and $\left(\partial^{2} / \partial \omega^{2}\right) \operatorname{Re} \tilde{W} \approx-5 / 2 \nu_{-}$, where $\omega_{0} \equiv V_{\star}^{\top} / r$ and $\alpha \equiv \sigma^{\prime}(40)(1-3 n / 2) \quad\left(\omega_{0}{ }^{2} \nu_{-} / \omega_{B i}{ }^{3}\right)$. To good approximation in $\left(\omega_{0} / \nu_{-}\right),\left(\gamma_{L} / \omega_{0}\right) \ll 1$ and for $n>0$, we find that in Eq. $(33 b) \delta \gamma(\infty) \approx$ $[(\partial / \partial \omega) \operatorname{Re} \hat{W}] \delta \Omega(\infty)$. Hence,

$$
\delta \Omega(\infty) \approx-\left(4 v_{-} / m_{\omega_{0}}\right) \gamma_{L}
$$

and

$$
\left|\Phi_{\infty}\right|^{2} \approx(16 / 3 n)\left(\gamma_{L} \nu_{-} / m^{2} \omega_{0}^{2}\right)=(32 / 3 n)\left(\frac{1}{4 \alpha}-v\right) \alpha
$$

For reversed-gradient profiles $(n<0)$, the frequency shift is positive in Eq. (33a); totally different values of $\delta \Omega(\infty)$ and $\left|\Phi_{m}\right|^{2}$ result. However, our fluid model omits the physical effects of reversed gradients in the linear electron driving term which exerts a significantly stabilizing influence [21]. Therefure, we w111 only consider equilibria for non-reversed-gradient profiles $(n>0)$ for which the frequency shift $\delta \Omega(\infty)$ is negative, proportional to the Iinear growth rate $\gamma_{L}$, and considerably larger than $\delta \gamma$ by a factor $4 \nu_{-} / m_{\omega_{0}}>\mid$.

The time-dependent solution of Eq. (32) is straightforward. We use $|\delta \gamma(t) / \delta \Omega(t)| \ll 1$ and $|(\partial \operatorname{Re} \tilde{W} / \partial \omega) \delta \Omega(t)| \gg\left|\left(\partial^{2} \operatorname{Re} \tilde{W} / \partial \omega^{2}\right) \delta \Omega(t)^{2} / 2\right|$ to simplify the mathematics. Solution of Eq. (32) is then obtained by differentiation with respect to time, separation of variables, and calculation of two elementary integrals. We find that

$$
\delta \gamma(t) / \gamma_{L}=\exp \left(2 \gamma_{L} t\right)\left[1-\operatorname{rexp}\left(2 \gamma_{L} t\right)\right]^{-1}
$$


and

$$
\delta \Omega(t) / \delta \Omega(\infty)=\left(\left|\Phi_{0}\right|^{2} /\left|\Phi_{\infty}\right|^{2} \exp \left[\int_{0}^{t} d t-2 \operatorname{Im}_{\omega}\left(t^{-}\right)\right]=-\delta \gamma(t) / \gamma_{L},\right.
$$

where $\Gamma \equiv-\left(\left|\Phi_{0}\right|^{2} /\left|\Phi_{\infty}\right|^{2}\right)\left(1-\left|\Phi_{0}\right|^{2} /\left|\Phi_{\infty}\right|^{2}\right)^{-1}$. Thus the nonlinear complex frequency shift and squared amplitude grow exponentially at rate $2 \gamma_{L}$ until just before saturation. We emphasize that saturation of the unstable wave in this case occurs for reasons much the same as for the equilibria established by three-wave interactions: mode coupling again provides for the formation of a balance between linear driving and dàmping forces.

4.2 Stability of Equilibria Established by Four-wave Interactions.

In this sub-section we investigate the linear stability of the equilibria constructed in the immediately preceding discussion. Consider a perturbation expansion $\Phi(x, y ; t) \equiv \Phi_{0}(x, y ; t)+\Phi_{1}(x, y ; t)$, where the equilibrium satisfies Eq. (28) with solutions described by Eqs. (33) and (34)

$$
\Phi_{0}(x, y ; t)=\left|\Phi_{\infty}\right| \exp \left[-i \Omega L t-i \delta \Omega(\infty) t+i k_{y} y\right] \sin \left(k_{x} x\right)+c . c .,
$$

and the perturbation satisfies Eq. (28) to first order in $\Phi_{1} / \Phi_{0}$

$$
\begin{gathered}
{[(\partial / \partial t)-W]\left(\Phi_{1}+\Phi_{0}^{2} \Phi_{1} / 2\right)-\eta_{j} V_{\star}^{\top}(\partial / \partial y) \Phi_{0}^{2} \Phi_{1}} \\
+2 \varepsilon^{-1}\left(1-\eta !\left(\omega_{0} / \nu_{-}\right) V_{\star}^{\top} r_{n} r\left[\left(\partial_{x} \Phi_{1} \partial_{y}-\partial_{y} \Phi_{1} \partial_{x}\right) \partial_{y} \Phi_{0}^{2}+2\left(\partial_{x} \Phi_{0} \partial_{y}-\partial_{y} \Phi_{0} \partial_{x}\right) \partial_{y}\left(\Phi_{0} \Phi_{l}\right)\right]=0\right.
\end{gathered}
$$

The same choice of boundary conditions is made here as for the equilibrium $\Phi_{1}\left(k_{x} x=0, \pi\right)=0$, and $\Phi_{1}\left(k_{y} y+2 \pi\right) \doteq \Phi_{1}\left(k_{y} y\right)$, where $k_{y}=\mathrm{mr}^{-1}, \mathrm{am}^{2}=1 / 2$, and $k_{x}=\pi k_{y}$. For $\Phi_{l}=\sum_{\ell, n} \Phi_{\ell n}(t) \exp \left(i_{\ell} r^{-1} y-i_{\omega_{\ell, n}} t\right) \sin \left(n k_{x} x\right)+c . c$. where $\omega_{\ell n} \equiv \omega_{L}\left(k_{y}^{\prime}=\ell r^{-1}, k_{x}^{\prime}=n k_{x}\right)$, Eq. (36) leads to an infinite matrix equation describing the coupling of the linear mode amplitudes $\Phi_{\ell n}$ via the equilibrium $\Phi_{0}$. 
We recall that all modes other than the principal mode of the equilibrium are assumed to be linearly damped and are consequently not spontaneously excited to significant amplitudes. We apply the arguments of Sec. 4.1 and therefor restrict our investigation of Eq. (36) to the consideration of only the nonlinear couplings which exactly satisfy the phase matching conditions Eqs. (29). This reduces the infinite matrix to diagonal form, viz.

$$
\left[-i \omega_{\ell n}-i \delta \omega^{-}-\tilde{W}\left(\omega, \ell r^{-1}, n k_{x}\right)-i(3 n / 2) \ell \omega_{0}\left|\Phi_{\infty}\right|^{2}\right] \tilde{\Phi}_{\ell n}=0,
$$

where $\omega=\omega_{\ell n}+\delta \omega^{-}$and we have Laplace-transformed in time $\partial / \partial t \rightarrow-i \delta \omega$. Recognizing that this equation has the same structure as Eq. (31), we conclude that the finite amplitude of the equilibrium $\Phi_{0}$ evidently induces a frequency shift in all the modes of the system. If this frequency shift is sufficient to nonlinearly destabilize any linearly stable mode in the system, then the equilibrium is unstable.

In order that there be only a single linearly unstable mode,

$$
\begin{aligned}
\gamma_{L}(m \pm 1) \approx \gamma_{L}-2 \omega_{0}{ }^{2} / \nu_{-} & \leq 0 \text { or } \\
0 & \leq \frac{\gamma_{L}}{\left(\omega_{0}^{2} / \nu_{-}\right)}=\frac{1}{4 v}-v \leq 2 .
\end{aligned}
$$

Because the mode frequency has been assumed to satisfy $\nu_{+} \ll m \omega_{0} \ll \nu_{-}$, where $\nu \equiv \nu_{+} \nu_{-} / \omega_{0}^{2}$ and $\nu_{+} \approx(1 / 2)\left(m_{e / m_{i}}\right)^{1 / 2} \nu_{-}=\theta\left(10^{-2}\right) \nu_{-}$for a deuterium plasma, we find that $O(0.1) \leq v \leq \boldsymbol{O}(10)$. Then Eq. (38) allows us to establish a condition on $\alpha$, viz. $\boldsymbol{\Theta}\left(10^{-2}\right)<\alpha<1$; and hence $m \leq 7$.

A slightly more severe condition on $\alpha$ is obtained from a consideration of the nonlinear shift of the sideband frequencies. We Taylor-series expand $\tilde{W}$ in Eq. (37) and employ the standard frequency orderings $\left(\omega_{0} / \nu\right),\left(\gamma_{L} / \omega_{0}\right) \ll<$ to obtain

$$
\delta \Omega^{-}=2(\ell / \mathrm{m}) \delta \Omega(\infty)
$$

and

$$
\left.\delta \gamma^{-} \approx \frac{\partial}{\partial \omega} \operatorname{Re} \bar{W}\right|_{\omega \ell n} \delta \Omega^{-}+\left.\frac{\partial^{2}}{\partial \omega^{2}} \operatorname{Re} \tilde{W}\right|_{\omega_{\ell n}} \delta \Omega^{-2} / 2,
$$


where $\delta \Omega(\infty)$ is" given by Eq. (34a). The most stringent condition on stability is obtained by considering the perturbed modes which are nearest sidebands of the equilibrium and which might sustain $\delta y^{-}>0, v i z, \ell=m \pm 1$ and $n=1$. From Eq. (27) we determine that $(\operatorname{Re} \tilde{W} / \partial \omega)=\left(\omega_{0} / \nu_{-}\right)( \pm 4-m / 4)$ and $\left(\partial^{2} \operatorname{Re} \tilde{W} / \partial \omega^{2}\right)=$ $\left(1 / v_{-}\right)[ \pm(4 / m)+(5 / 2)]$ for $\ell=m \pm 1$. Then Eqs. (39) give $\delta \Omega^{\prime}=2(m \pm 1)$ $x \delta \Omega(\infty) / m \approx-8 \gamma_{L \nu_{-}} / m_{\omega_{0}}$ for $m \gg 1$ and $\delta \gamma^{-} \approx\left[-2 \pm(32 / m] \gamma_{L}\right.$. From $\operatorname{Im}_{\omega_{\ell n}}=\gamma_{L}(\ell=m \pm 1) \approx \gamma_{L}-2 \omega_{0}{ }^{2} / \nu_{-}$, the net growth rate for two sidebands becomes for $m>1$

$$
\text { Ilil } \approx-2 \omega_{0}^{2} / \nu+[ \pm(3 z / m)-1] \gamma_{L},
$$

which must be negative for stability.

The lower sideband is evidently further stabilized by the nonlinear frequency shift. However, for $m<32$ the upper sideband can be destabilized for linear growth rates $\gamma_{L}$ sufficientily large. We recall that $\gamma_{L}=\left(\omega_{0}^{2} / \nu_{-}\right)$ $x\left[(4 \alpha)^{-1}-\nu\right]$, which using Eq. (40) allows us to express the condition $\operatorname{Im}_{\omega}<0$ equivalently as

$$
\frac{\gamma_{L}}{\left(\omega_{0}{ }^{2} / \nu_{-}\right)}=\frac{m^{2}}{2} \quad-v<\frac{2 m}{32-m},
$$

where $1 \ll m<32$ and $\alpha \mathrm{m}^{2} \approx 1 / 2$. Of course, the linear stability of the sidebands already demands that $m \leq 7$. For $\theta(0.1) \leq v \leq \theta(10)$, as consequence of Eq. (41) we determine that $m \leq 5, \alpha \geq 0.0<$, and $\gamma_{L}\left(\omega_{0}{ }^{2} / \nu_{-}\right)^{-1}<O(1 / 2)$ to guarantee the nonlinear stability of the upper sideband.

5. Cross-field Transport and Scaling for Tokamak Plasmas

5.1 Transport Coefficient for Radial Flux

This section presents a calculation of the anomalous transport arising from the trapped-ion mode. We:compare the transport levels for saturation due to coherent mode coupling in two dimensions, due either to three-wave or four-wave interactions, with the transport levels predicted by Kadomtsev and Pogutse $[11,12]$. 
The coherent radial transport of particles is given by the flux $\left\langle n_{s} v_{s} \cdot \hat{x}\right\rangle$, where the brackets indicate an average over the poloidal angle. Quasineutral$i$ ty and the dominance of the Ex $\underline{B}$ contribution to the velocity of each species account for the equality of $\left\langle n_{i} \underline{V}_{i} \cdot \hat{x}\right\rangle \approx\left\langle n_{e} v_{e} \cdot \hat{x}>\right.$ to lowest significant order. The poloidally averaged electron flux is given by

$$
<n_{e} V_{e} \cdot \hat{x}>=(2 \pi r)^{-1} \int_{0}^{2 \pi r} d y\left(n_{e} V_{e} \cdot \hat{x}\right)=(2 \pi r)^{-1} \int_{0}^{2 \pi r} d y\left(n_{e} T_{e}^{T} \cdot \hat{x}\right) ;
$$

the circulating electrons do not contribute to the average radial flux because they respond adiabatically. The poloidal average is equivalent to a time average over many oscillation periods. Because of poloidal periodicity, the diamagnetic fluid velocity produces no average flux to all orders in $\Phi$.

Eq. (12) gives an expression for $n_{e}^{\top}$ accurate to $\sigma\left(\Phi^{3}\right)$. As argued in deriving Eq. (10), for purposes of calculating the particle fluxes the fluid velocities can be replaced by the $\underline{E} \underline{B}$ drift: $\underline{V}_{s}+\hat{c} \hat{Z} \times \underline{\nabla} \phi / B$. The nonlinear contributions to $n_{s}$ were found to be much more important than the ponderomotive contributions to $\underline{V}_{S}$. The transport coefficient is defined by $D=-\left\langle n_{s} v_{s} \cdot \hat{x}>\right.$ $x\left(d n_{0} / d x\right)^{-1}$, which leads to

$$
\begin{aligned}
D \approx-r_{n}(C T / e B) & <\left\{n_{0}^{-1}\left(D / \nu_{-} D t\right)\left[2 n_{0}\left(1-\varepsilon^{1 / 2}\right)\left(\Phi+\Phi^{3} / 6\right)-\delta n\right]\right. \\
& \left.+\left(\nu_{+} / \nu_{-}\right)\left[2 \Phi-\left(\delta n_{1} n_{0}\right)+\Phi^{3} / 3\right]\right\} \partial \Phi / \partial y>.
\end{aligned}
$$

To lowest order $(D / D t)+(\partial / \partial t)$ and $\partial \Phi / \partial t \approx-V_{\star}^{\top} \partial \Phi / \partial y$, where use has been made of the frequency ordering, $\varepsilon \ll 1$, and $\Phi \ll 1$. Hence, Eq. (42) gives

$$
\mathrm{D} \approx\left(\frac{\mathrm{cT}}{\mathrm{eB}}\right)^{2}{\frac{\mathrm{c}}{v_{-}}}^{1 / 2}\left\langle\left(\frac{\partial \Phi}{\partial y}\right)^{2}\right\rangle=\frac{4 \mathrm{~V}_{\star}^{\mathrm{T}}{ }^{2} r_{n}{ }^{2}}{\varepsilon^{1 / 2} v_{-}}\left\langle\left(\frac{\partial \Phi}{\partial y}\right)^{2}\right\rangle
$$

The next higher order terms in $\Phi$ are smaller by relative order $\left|\Phi / \varepsilon^{1 / 2}\right|$ if three-wave interactions are effective; otherwise the next higher 
order terms are $\left(\Phi^{4}\right)$, or of relative order $|\Phi|^{2}$ compared to those retained.

Kadomtsev and Pogutse have estimated a transport coefficient when saturation is provided by incoherent, turbulent processes, which is given by.

$$
D_{K P} \sim \varepsilon^{5 / 2}\left(\frac{{ }^{\top T}}{e} \frac{e}{e B r_{n}}\right)^{2} \frac{1}{4 \nu_{e}}=\frac{v_{*}^{\top} 2}{\nu_{-}} \varepsilon^{1 / 2}
$$

Their calculation assumes that there are many unstable modes, i.e. the plasma is far from marginal stability.

In order to compare the Kadomitsev-Pogutse estimate with our general expression, Eq. (43), the quantity $<(\partial \Phi / \partial y)^{2}>$ must be evaluated. In the preceding sections appear calculations of the nonlinear saturation of the trappedion mode for plasmas having moderate-sized $\alpha$, viz. $10^{-2} \leq \alpha<1$, either well above marginal stability or so close to marginal stability that only a single mode is unstable. Appropriate for the case far from marginal stability $\left(\nu_{+} \rightarrow 0\right)$ in which three-wave interactions are dominant, it was previously calculated [15] that nondispersive, one-dimensional two-mode equilibria have a transport cocfficient given by

$$
\quad 0 \sim \frac{2}{\varepsilon} \frac{v_{x}^{T}{ }^{\prime 2}}{v_{-}} \frac{r_{n}^{2}}{r^{2}}\left[\frac{\varepsilon}{(1-n)^{2}}{ }^{\frac{\omega_{0}^{2}}{2}} \frac{1}{4 \alpha^{2}}\right]
$$

The quantity in the square brackets in Eq. (44) is llie saturated valuc of $r^{2}<(\partial \Phi / \partial y)^{2} ;$; values of this quantity for disperstve eyuilitria including two-dimensiona $i$ effects can be calculated from Eqs. (22-25) and scale quite differently. Numberical examples of the prediction of $r^{2}<(\partial \Psi / \partial y)^{2} ;$ made by our model in various parameter regimes are presented in the final sub-section.

Near marginal stability we have demonstrated that a single unstable mode can saturate its own growth by producing a frequency shift which allows a balance to be established between electron driving and jon damping forces. That 
these forces are separately finite is responsible for there being energy flow and particle transport in the system even at saturation. From Eqs. (34b) and (43), the transport coefficient for the saturation due to the self-coupling of a single marginally unstable mode is given by

$$
D \sim \frac{2}{\varepsilon^{1 / 2}} \frac{v_{\star}^{\top ?}}{v_{-}} \frac{r_{n}^{2}}{r^{2}}\left[\frac{32}{3}\left(\frac{1}{4 \alpha}-v\right)\right]
$$

In Ref. 15 a fairly detailed comparison of the Kadomtsev-Pogutse transport coefficient and that for one-dimensional, nondispersive two-mode equilibria [ Eq. (44).] was presented. Profiles typical of the Princeton Large Torus (PLT) were considered. It was found that the transport accompanying coherent three-wave coupling could be substantially smaller than the Kadomtsev-Pogutse estimate over much of the plasma cross-section. Very roughly, for $\left(r_{n} / r\right)=\boldsymbol{O}(1)$, moderate values of $\alpha$, and $4 \alpha \nu<1$, Ref. 15 determined that

$$
D / D_{K P} \sim\left[2 /(1-n)^{2}\right]\left(\omega_{0}^{2} / \nu_{-}\right)\left(4 \alpha^{2}\right)^{-1}<1 .
$$

The inclusion here of radial effects and especially the increased coupling provided by $\beta \neq 0$ in Eq. (15) reduces the saturated amplitudes and consequently improves the transport picture by reducing $D / D_{K P}$.

\subsection{Scaling and Summary}

In this section we conclude our discussion with a few remarks on the scaling of the values of the saturated amplitudes, a review of the more important assumptions made by our model and its limitations, and a brief summary of the important results. A detailed consideration of the dependence of crucial parameters on the experimental conditions typical of a large tokamak, e.g. PLT, appears in Ref. 15 and sha11 not be duplicated here.

$\Lambda$ simple figure of merit in calibrating the value of the saturated mode amplitude(s) is the value of $e_{\phi} / \varepsilon T$. When for a single wave this exceeds unity 
a trapped ion resonantly interacting with a wave can be electrostatically detrapped by simple coherent acceleration due to the parallel electric field, if its bounce length is appreciable compared to $2 \pi / k_{11} \sim$ connection length $\doteq q R$. Investigations of electrostatic detrapping in the presence of turbulence $[9,13]$ or for a single wave with stochastic effects included [19] have lead to considerably lower estimates of $e_{\phi} / \varepsilon T$ at saturation by factors typically of order ten to twenty.

The analysis in Ref. 15 of one-dimensional, non-dispersive equilibria established by three-wave interactions gives a result

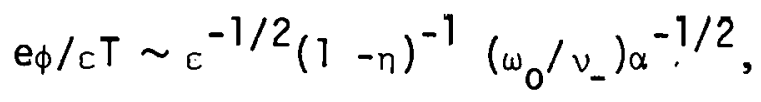

where for validity of the model $\left(\omega_{0} / \nu_{-}\right), \varepsilon<1 ; n<2 / 3$; and $Q\left(10^{-2}\right)<\alpha<1$. For typical tokamak parameters admitting the trapped-ion instability, the expression for $e_{\phi} / \varepsilon T$ in Eq. (46). could possibly be of order unity. We now numerically demonstrate how the inclusion of radial effects can dramatically alter this estimate.

For purposes of numerical evāluation of Eqs. (22 - 25) we chuose the following basic parameters: $\nu_{-} / \omega_{0}=20.4, n=1 / 2, \varepsilon=0.1, \omega_{0} / \omega_{B i}=0.0612$, $m_{i} / m_{e}=3600$ and $\delta_{R} \equiv\left(\nu_{-} / \omega_{n}\right)\left(\rho_{i}{ }^{2} q^{2} / \varepsilon r^{2}\right)=0.04$. This gives $\alpha=A^{-}(1-3 n / 2)$ $x\left(\omega_{0} / \omega_{B j}\right)^{2}\left(\nu_{-} / \omega_{B i}\right)=0.04$ for $A^{-} \equiv 40[4,5]$ and $\delta_{U}=8(1-n)\left(\nu_{-} / \omega_{0}\right)$ $x\left(\omega_{0} / \omega_{B i}\right)^{2}=0.306$. The important parameter a scales according to $\alpha=5.5 \times 10^{-4}(1-3 n / 2) q^{3} \varepsilon^{-7 / 2} R(\mathrm{~cm}) r_{n}(\mathrm{~cm})^{-2} n\left(10^{14} \mathrm{~cm}^{-3}\right) B(50 \mathrm{kG})^{-2} T(\mathrm{keV})^{-1}$.

In Table I we consider the influence on the equilibrium amplitudes of various parameter choices for $\beta=2(1-n)^{-1}\left(\omega_{0} / \nu_{-}\right)\left(r_{n} / r\right)$ and the level of dispersion. Our choice of parameters is meant only to be illustrative, but is consistent with the usual orderings, and ensures that $\operatorname{Re} \tilde{W}_{11}>0$, but that $\operatorname{ReW}_{02}$, $\operatorname{Re}_{21}, \operatorname{ReW}_{12}<0$. 
The complex values of the Fourier amplitudes $\tilde{e}_{m n} / \varepsilon T \equiv \varepsilon{ }^{1 / 2}(1-n)^{-1}$ $\left(\omega_{0} / \nu_{-}\right)\left(e a_{m n} / \varepsilon\right)$ and the group velocity $v_{g} / v_{*}^{\top} \equiv\left(\omega_{0} / \nu_{-}\right) u$ are tabulated in Table I. The cases for which $\beta=10^{-6}$ correspond closely to the one-dimensional two-mode equilibria $(\beta=0)$ except for geometrical correction factors of order unity arising from the simusoidal variation in $x$. Dispersion tends to inhibit effective coupling of the fundamental mode $\tilde{\phi}_{17}$ to the $\tilde{\phi}_{21}$ mode and hence force the saturated amplitudes from $\mathrm{e}_{11} / \varepsilon T \leq 1$ to $\mathrm{e}_{11} / \varepsilon T z 1$. For the $\beta$-dominated cases, $\beta=0.1$, the coupling of the fundamental to $\phi_{02}$ is more important than the coupling to $\tilde{\phi}_{21}$ and is much less influenced by dispersion for these parameter choices. The table makes obvious the dramatic reduction in saturated amplitudes, $\tilde{e}_{17} / \varepsilon T \sim 0.02$ for these parameters, produced by the enhanced coupling when $\beta \neq 0$.

Table I. Two-dimensional equilibrium amplitudes and group velocity $v_{g}$ established by three-wave interactions for $\varepsilon=0.1, \eta=1 / 2, \nu_{-} / \omega_{0}=20.4$, $\omega_{0} / \omega_{B i}=0.0612$, and $\rho_{i} q / \varepsilon^{1 / 2} r=0.044$

\begin{tabular}{|c|l|l|l|c|l|l|}
\hline$\beta$ & $\delta_{B}$ & $\delta_{U}$ & $\tilde{e}_{n} / \varepsilon T$ & $\tilde{e}_{21} / \varepsilon T$ & $\tilde{e}_{02} / \varepsilon T$ & $V_{g} / V_{*}^{T}$ \\
\hline \hline $10^{-6}$ & 0 & 0 & 0.473 & $-i .062$ & $-0.835 \times 10^{-4}$ & 0 \\
\hline $10^{-6}$ & 0.04 & 0.306 & 1.44 & $0.149-i .0416$ & $-0.775 \times 10^{-3}$ & 0.342 \\
\hline 0.1 & 0 & 0 & 0.0237 & $-i .155 \times 10^{-3}$ & -0.0209 & 0 \\
\hline 0.1 & 0.04 & 0.306 & 0.0237 & $0.403 \times 10^{-4}-i .113 \times 10^{-4}$ & -0.0210 & 0.389 \\
\hline
\end{tabular}

For plasmas very close to marginal stability we have calculated equilibria established by the self-coupling of a single, marginally unstable wave via a four-wave interaction. Using Eq. (34b) we calculate that at saturation

$$
\mathrm{e} \phi / \varepsilon \mathrm{T} \sim 8 \varepsilon^{-1}(\alpha / 3 n)^{1 / 2}\left(\frac{1}{4 \alpha}-v\right)^{1 / 2} \text {, where } n>0 \text {. }
$$


We recall that the simple nonilinear stability arguments presented in Sec. 4.2

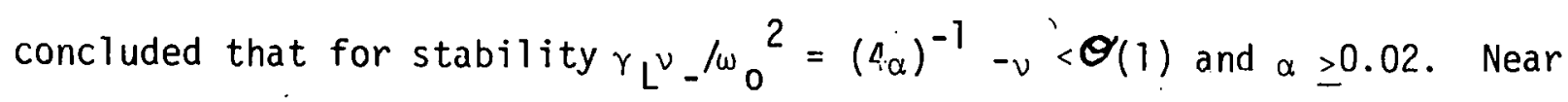
linear marginal stability the value of $e_{\phi} / \varepsilon T$ can evidently be very small, i.e. $\mathrm{e}_{\phi} / \varepsilon T \propto\left(\gamma_{L} \nu_{-} / \omega_{0}^{2}\right)^{1 / 2} \rightarrow 0$ as $\gamma_{L} \rightarrow 0$.

We caution the reader not to take the exact numerical predictions of our model too seriously in view of the many approximations made. The augmented Kadomtsev-Pogutse fluid equations give only a simple semi-quantitative model of the trapped-ion mode. A more rigorous kinetic treatment of the nonlinear ievolution of the mode and accompanying computer simulations are needed in our opinion.

The scaling with tokamak parameters of most of the important assumptions in our model has been previously discussed [15]. However, we emphasize that validity of the model demands that $\omega_{0} \ll \omega_{B i}, \omega \ll k_{11}\left(T_{s} / m_{s}\right)^{1 / 2}$, $\varepsilon \ll 1$, and $\nu_{+} \ll \omega_{0} \ll \nu_{-}$to justify the Kadomtsev-Pogutse fluid equations, $\left|\delta x_{B}+\delta x_{T}+\delta x_{U}\right| \ll 2$ to guarantee that the kinetic effects added are only weakly perturbative, and $\Phi \equiv e_{\phi} / T \ll 1$ to ensure convergence of the perturbation expansion in powers of $\Phi$. These conditions conspire to make the mudel in which three-wave interactions are effective valid only in a narrow regime of parameter space: $\nu_{-} / \omega_{\mathrm{Bi}} \sim \theta(1)$ and of the parameter $\alpha$, evaluated algebrdically in Eq. (47) and numerically in [15], $10^{-3}<\alpha<1$. This is based on a calculation of the effects of dispersion arising from untrapped ions which is referred to in Ser. 3.1. The model calculations in which four-wave interactions are dominant are valid near maryinal stability, $1 . c$. when $(1 / 4 a)-v<\theta(1)$ and $\left(10^{-2}\right)<\alpha<i$.

We have made no estimates of the limits determined by use of linear kinetic theory in describing the effects of finite ion banana-width excursions, thermal and resonant corrections to the circulating ion response, and trappedion Landau damping. Nonlinear calculations of electrostatic detrapping 
$[9,13,18]$ presumably offer estimates of what levels of electrostatic potential are necessary to produce significant nonlinear orbit modifications. However, in view of the lack of consensus among the theories of electrostatic detrapping and the approximate nature of these theories and of our own mode-coupling theory, precise quantitative comparison is premature. Nevertheless we can conclude that the estimates of saturation by mode.coupling seem to be quite competitive with the lowest estimates made by the theories of electrostatic detrapping.

We have thus extended a mode-coupling theory of the saturation of the trapped-ion mode based on the augmented Kadomtsev-Pogutse fluid equations to include variations in the two dimensions corresponding to the plane perpendicular to the magnetic field. Important linear kinetic effects have been systematically added. The nonlocal effect that magnetic shear has on circulating-ion Landau damping has been very simply incorporated in the limit that the spacing between mode rational surfaces of the most linearly unstable mode is much shorter than any characteristic scale length of the basic plasma parameters. We have offered a more general derivation of a fundamental equation of evolution for the electrostatic potential Eq. (13) than has previously appeared $[10,15]$. Simple time-dependent and steady-state approximate solutions of this equation have been obtained analytically.

Equilibria.established by three-wave interactions have been shown to potentially result in significantly smaller saturated amplitudes and weaker concomitant particle transport as compared with the predictions of previous onedimensional theory [15]. The transport coefficient can therefore be substantially reduced below the Kadomtsev-Pogutse estimate. Our two-dimensional equilibria illustrate the need for an energy sink at short radial wavelengths to counterbalance the effects of fluid steepening. Following the dild lysts of Gladd and Ross, we have suggested that inclusion of the i inear effects due to 
finite ion banana-width excursions provides the necessary stabilization. We have found that a stability analysis of these equilibria is inconclusive and speculate that this might be due to the approximate nature of the equilibria.

Near linear marginal stability three-wave interactions cease to be effective and the $\Theta\left(\Phi^{3}\right)$ terms in the fundamental equation must be considered. The time-dependent approach toward an equilibrium produced by the self-coupling of a single linearly unstable mode via a resonant four-wave interaction has been analytically calculated. We have also determined for what plasma parameters these equilibria are stable. The particle transport at saturation is found to scale as the linear growth rate and $e \phi / \varepsilon T$ scales as the square root of the growth rate, both of which become vanishingly small as linear marginal stability is approached. 


\section{ACKNOWLEDGEMENTS}

We gratefully acknowledge the many contributions made by Drs. J. A. Krommes and M. N. Rosenbluth and their encouragement. We are also indebted to Drs. C. Oberman, G. Rewoldt, and D. W. Ross for numerous useful discussions.

This work was supported by the U. S. Energy Research and Development Administration Contract Nos. E(11-1)-3073 and W-7405-Eng-48. 


\section{REFERENCES}

\# Present ạdị rẹs

[1] Kadomtsev., B.B., Pogutse, O.P., Zh.Ehksp. Teor. Fix. 51 (1966) 1734 [Sov. Phys. - JETP 24 (1967) 1172].

[2] Kadomtsev, B.B., Pogutse, 0.P., Dok1. Akad. Nauk SSSR 186 (1969) 553 [Sov. Phys. - Dok1. 14, (1969) 470].

[3] Galeev, A.A., Sagdeev, R.Z., Dok1. Akad. Nauk SSSR 180 (1968) 839 [Sov. Phys. - Dok1. 13 (1968) 562].

[4] Rosenbluth, M.N.., Ross, D.W., Kostomarov, D.P., Nucl. Fusion 12 (1972) 3 .

[5] Tang, W.M., Nucl. Fusion 13 (1974) 883.

[6] Gladd, N.T., Ross', D.W., Phys. Fluids 16 (1973) 1706.

[7] Tang, W.M., Phys. Fluids 17 (1974) 1249.

[8] Dobrowolny, M., Ross, D.W., Phys. Fluids 18 (1975) 717.

[9] Jabion, C., Phys. Rev. Lett. 28 (1972) 880.

[10」 LaQuey, R.E., Mahajan, S.M., Rutherford, P.H., Tárÿ, W.M., Fhys. Rev. Lett. $31(1975) 391$.

[11] Kadomtsev, D.B., rogutsc, O.P., in Reviows of Plassma Physirs. (Leontovich, N., td.). $\underline{5}$, Consultants Bur'edu, New Yürk (1970).

[12] Kadomtsev, B.B., Pogutse, O.P., Nucl. Fusion 11 (1971) 67.

[13] Ehst, D.A., Ph.D. Thesis, Mass. Inst. of Tech., (1976).

[14] Horton, W., Ross, D.W., Tang, W.M., Berk, H.L., Frieman, E.A., LaQuey, R.E., Luveldie, R.V., Mahajan, S.M., Rosenbluth, M.N., Rutherford, P.H., in Plasma Physics and Controlled Nuclear Fusion (Proc. 5th Int. Cont., Tokyo, 1974) 1, IAEA, Vienna (1975) 541; Lovelace, R.V., Tang, W.M., Bul1. Am. Phys. Soc. 19 (1974) 867. 
[15] Cohen, B.I., Krommes, J.A., Tang, W.M., Rosenbluth, M.N., Nucl. Fusion 16 (1976) 971 .

[16] Saison, R., Wimmel, H.K., in Plasma Physics and Controlled Nuclear Fusion (Proc. 6th Int. Conf. Berchtesgaden, 1976) , IAEA, Vienna (1977).

[17] Ross, D.W., Horton, C.W., Phys. Rev. Lett. $\underline{28}$ (1972) 484.

[18] Tang, W.M., Adam, J.C., Ross, D.W., Phys. Fluids 20 (1977) 613;

Tagger, M., Laval, G., Pellat, R., (to be published in Nucl. Fusion).

[19] Smith, G.R., Phys. Rev. Lett. 38 (1977) 970.

[20] Nishikawa, K., J. Phys. Soc. Japan 24 (1968) 916; J. Phys. Soc. Japan 24 (1968) 1152.

[21] Tang, W.M., Rutherford, P.H., Furth, H.P., Adam, J.C., Phys. Rev. Lett. 35 (1975) 660 . 


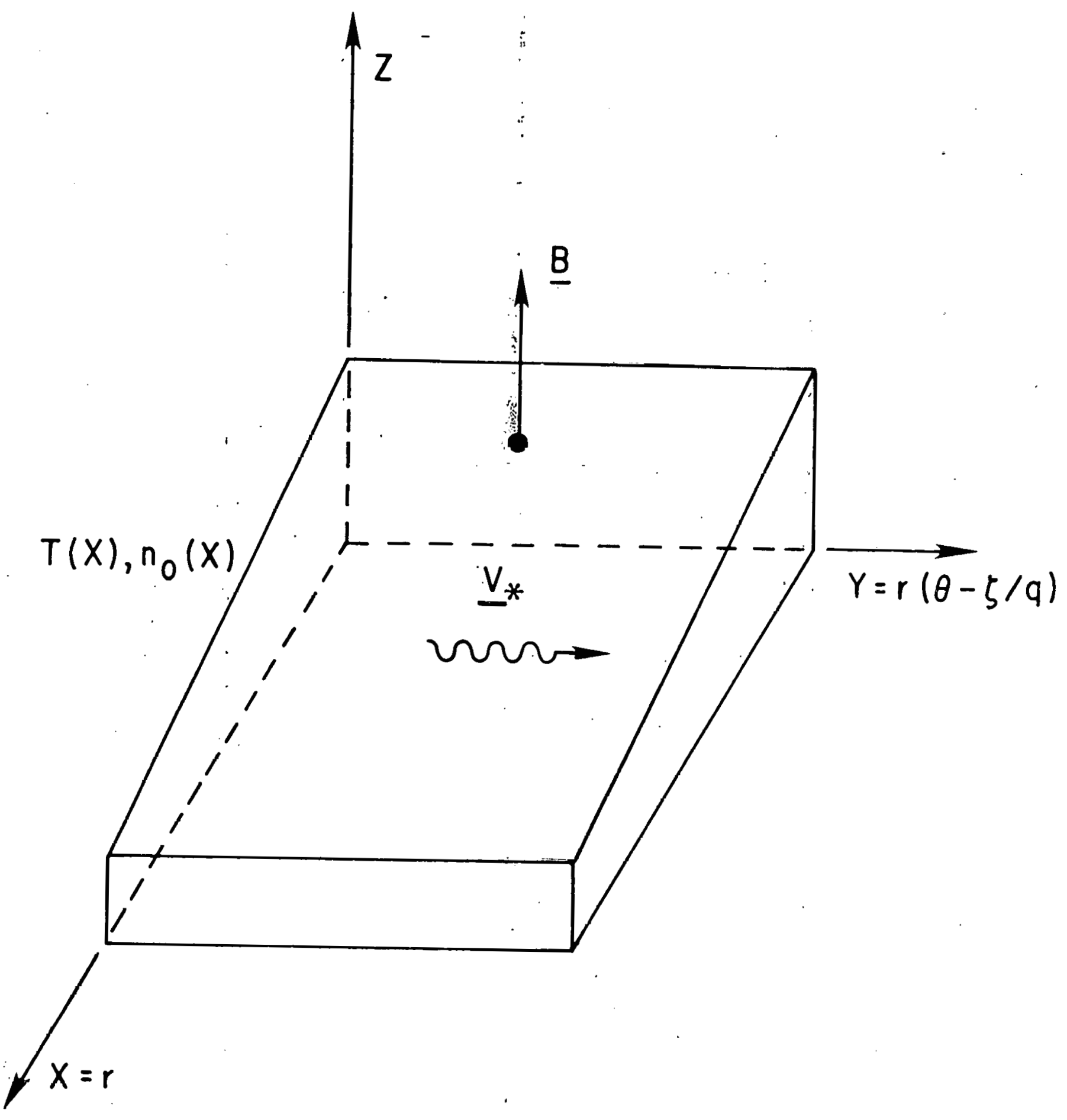

Fig. ' 1

Fig. 1 Slab coordinates showing mutually orthogonal density and temperature gradients $\nabla n_{0}(x)$ and $\nabla T(x)$, magnetic field $\underline{B}$, and diamagnetic drift velocity $\underline{V}_{\star}$. 


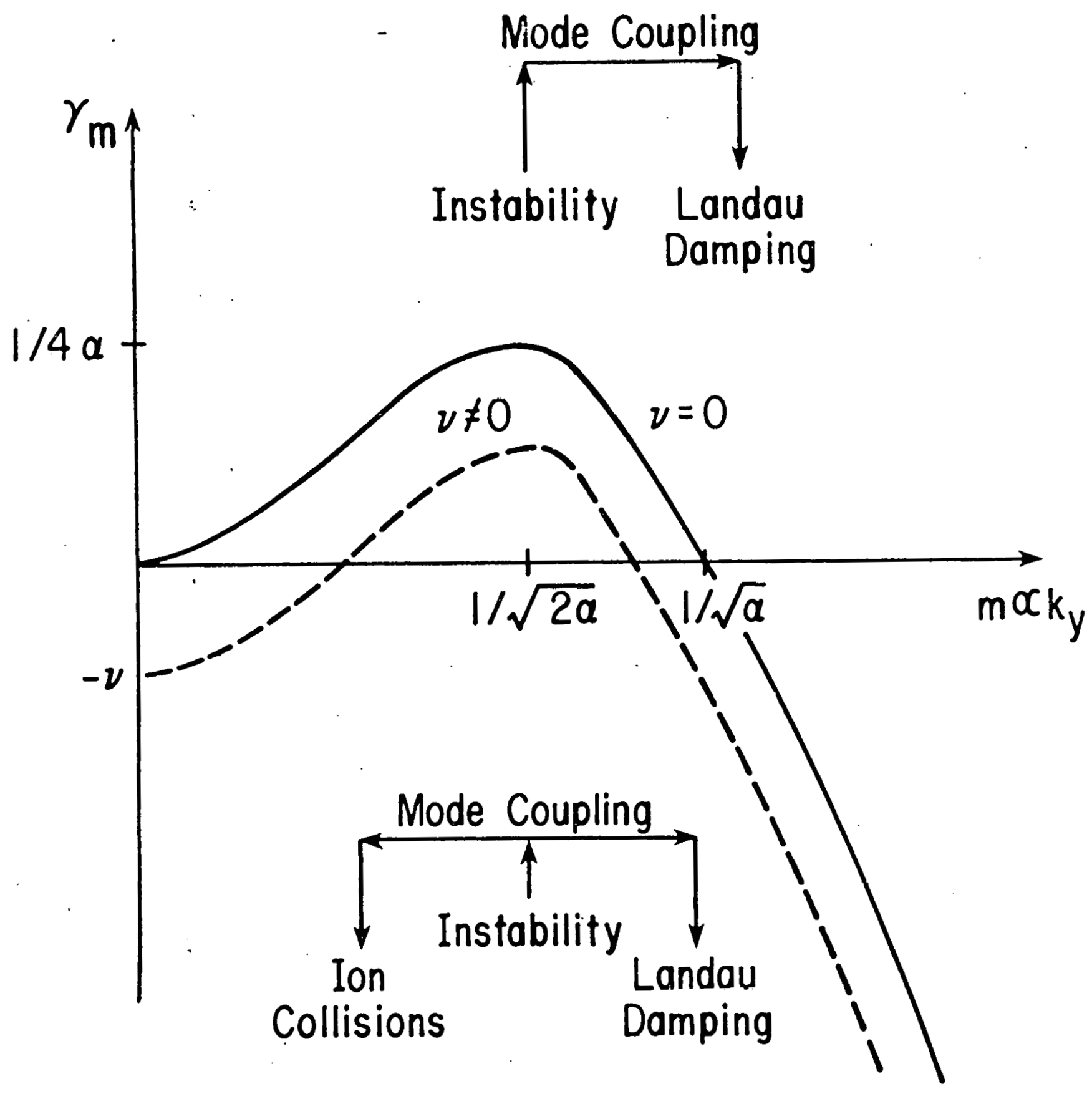

Fig. 2

Fig. 2 Diagram of the non-dimensional linear growth rate $\gamma_{m}=m^{2}$ - $a m^{4}-v$ as function of mode number $m, k_{y}=m / r$, with parameter $v \equiv v_{+} v_{-} / \omega_{0}^{2}$. The flow of wave energy is schematicaliy presented. 

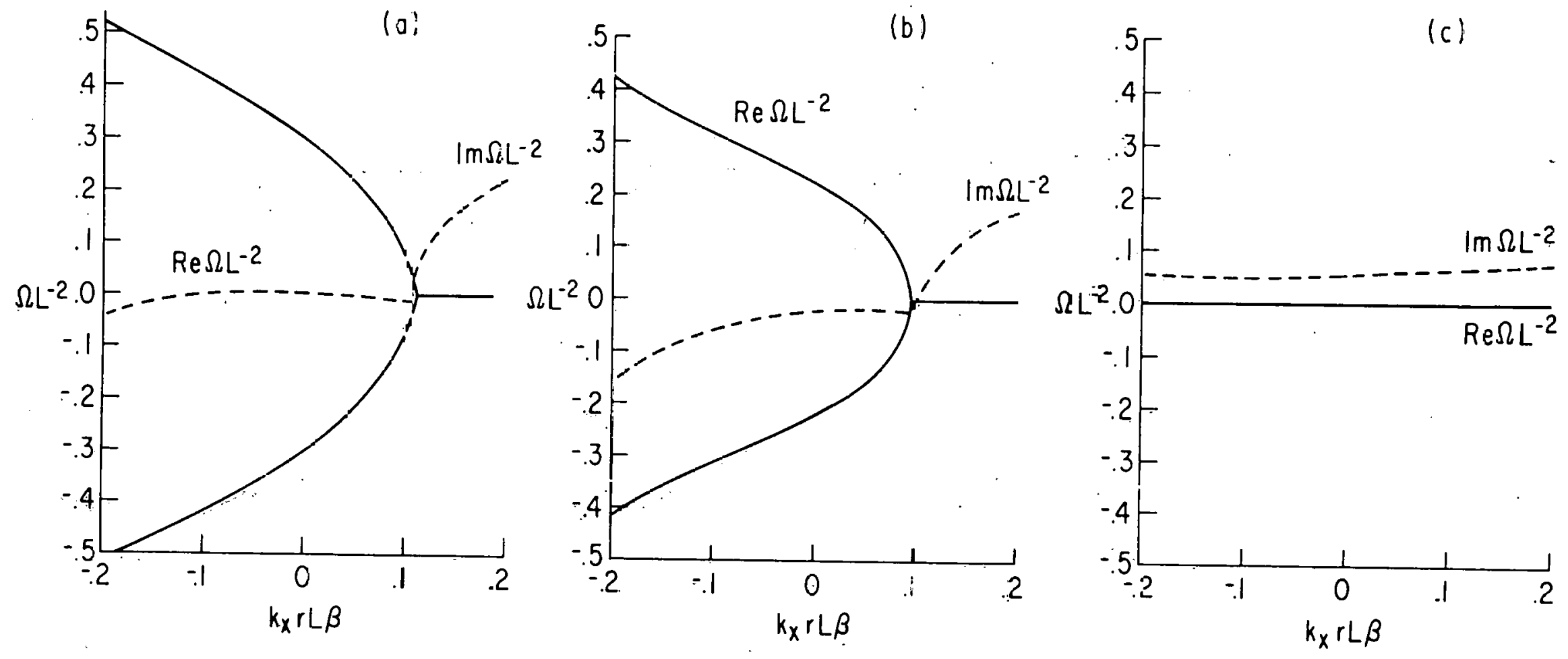

Fig. 3

Fig. 3

Frequenc: eigentalues $s_{c}$ in the drift-wave frame for linearly perturbed nondispersive two-mode equilibria as function of $k_{x} r L B$. The perturbation $\hat{c} \psi=\exp \left(-i_{\Omega \tau} i \exp \left(i k_{x} r_{\rho} i \sum_{n^{\prime}} a_{m+n} L \exp [i(n+n-L) \xi]-c . c\right.\right.$. is characterized by $m=1$, and the equilibrium $\psi_{0}=a_{L} \exp (i L \xi)+\exists_{2 L} \exp (i 2 L \xi)+c . c$. is parameterized sy $L=8$ and $(\hat{c}) \alpha L^{2}=.56,(b) \alpha L^{2}=.64$, and $(c) \alpha L^{2}=.72$. 


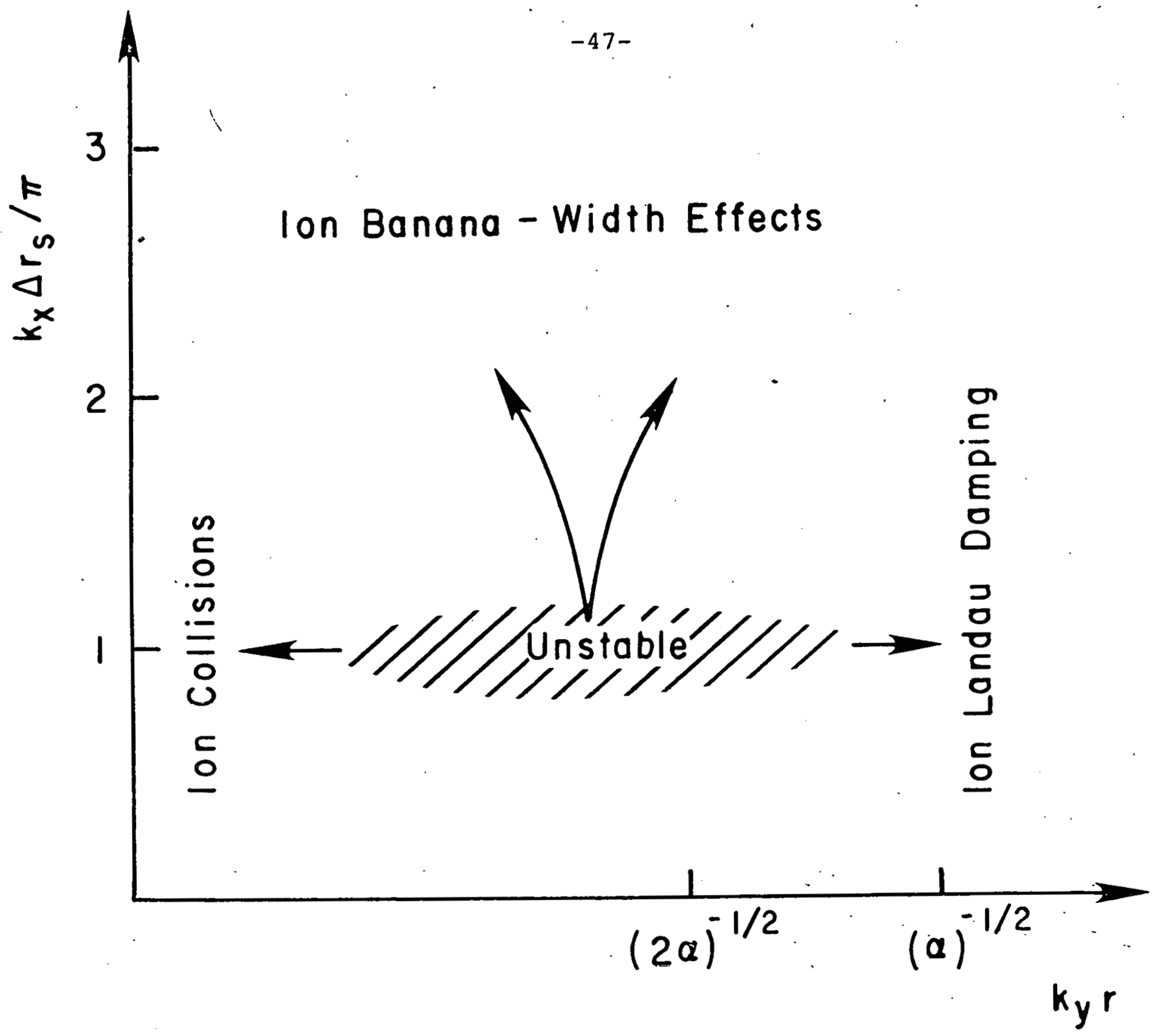

Fig. 4

Fig. 4 Schematic of the flow of energy due to mode coupling in twodimensional Fourier space $\left(k_{x}, k_{y}\right)$ from unstable modes to modes stabilized by ion collisions, ion Landau damping, and effects associated with ion banana-width excursions. The quantity $\Delta r_{s}=\left(k_{y} r d \text { ln } q / d r\right)^{-1}$ is associaled with the value of $k_{y}$ for the principal unstable mode. 